\title{
Urinary biomarkers may provide prognostic information for subclinical acute kidney injury after cardiac surgery
}

Christian Albert, MD, ${ }^{\mathrm{a}, \mathrm{b}}$ Annemarie Albert, MD, ${ }^{\mathrm{a}, \mathrm{b}}$ Johanna Kube, MD, ${ }^{\mathrm{c}, \mathrm{d}}$ Rinaldo Bellomo, MD,

Nicholas Wettersten, MD, ${ }^{\mathrm{f}}$ Hermann Kuppe, MD, ${ }^{\mathrm{g}}$ Sabine Westphal, MD, ${ }^{\mathrm{h}}$ Michael Haase, MD,,${ }^{\mathrm{a} b}$ and Anja Haase-Fielitz, PharmD ${ }^{\mathrm{i}, \mathrm{j}}$

\section{ABSTRACT}

Objective: This study aimed to determine the biomarker-specific outcome patterns and short-and long-term prognosis of cardiac surgery-asoociated acute kidney injury (AKI) identified by standard criteria and/or urinary kidney biomarkers.

Methods: Patients enrolled $(\mathrm{N}=200)$, originated a German multicenter study (NCT00672334). Standard risk injury, failure, loss, and end-stage renal disease classification (RIFLE) criteria (including serum creatinine and urine output) and urinary kidney biomarker test result (neutrophil gelatinase-associated lipocalin, midkine, interleukin 6 , and proteinuria) were used for diagnosis of postoperative AKI. Primary end point was acute renal replacement therapy or in-hospital mortality. Long-term end points among others included 5-year mortality. Patients with single-biomarker-positive subclinical AKI (RIFLE negative) were identified. We controlled for systemic inflammation using C-reactive protein test.

Results: Urinary biomarkers (neutrophil gelatinase-associated lipocalin, midkine, and interleukin 6) were identified as independent predictors of the primary end point. Neutrophil gelatinase-associated lipocalin, midkine, or interleukin 6 positivity or de novo/worsening proteinuria identified $21.1 \%, 16.9 \%, 30.5 \%$, and $48.0 \%$ more cases, respectively, with likely subclinical AKI (biomarker positive/RIFLE negative) additionally to cases with RIFLE positivity alone. Patients with likely subclinical AKI (neutrophil gelatinase-associated lipocalin or interleukin 6 positive) had increased risk of primary end point (adjusted hazard ratio, 7.18; 95\% confidence interval, 1.52-33.93 $[P=.013]$ and hazard ratio, $6.27 ; 95 \%$ confidence interval, 1.12-35.21 $[P=.037])$, respectively. Compared with biomarker-negative/ RIFLE-positive patients, neutrophil gelatinase-associated lipocalin positive/ RIFLE-positive or midkine-positive/RIFLE-positive patients had increased risk of primary end point (odds ratio, 9.6; 95\% confidence interval, 1.4-67.3 $[P=.033]$ and odds ratio, $14.7 ; 95 \%$ confidence interval, 2.0-109.2 [P=.011], respectively). Three percent to $11 \%$ of patients appear to be influenced by single-biomarkerpositive subclinical AKI. During follow-up, kidney biomarker-defined short-term

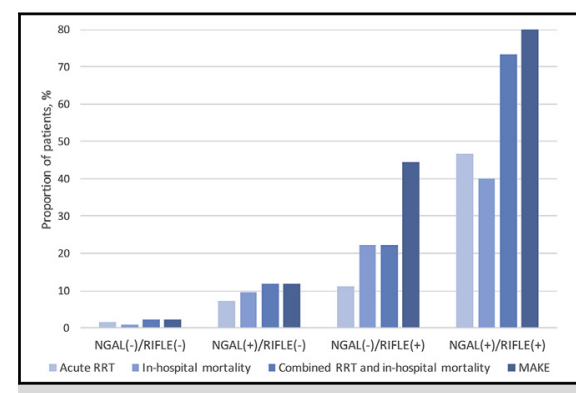

Proportion of patients with defined end points according to NGAL/RIFLE status. Biomarker (NGAL) positivity associates with worse patient outcome irrespective of RIFLE status.

\section{Central Message}

Positive urinary kidney biomarker test result after cardiac surgery carries prognostic information regardless of whether renal function will acutely decline (positive RIFLE criteria) or not.

\section{Perspective}

Information about subclinical acute kidney injury (AKI) is detectable with urine tests readily available in routine clinical practice. Recognition of subclinical AKI carries prognostic information and may contribute to refined clinical risk assessment. Single biomarker-positive, subclinical AKI deserves further investigation as a novel pathophysiologic AKI phenotype.

See Editorial Commentary page 2453.

See Editorial page 2439.

\footnotetext{
From the ${ }^{\text {a}}$ Medical Faculty Otto-von-Guericke University, Magdeburg, Germany; ${ }^{\mathrm{b}}$ Diaverum Deutschland, Potsdam, Germany; ${ }^{\mathrm{c}}$ Clinic of Nephrology and Hypertension, Diabetes and Endocrinology, Otto-von-Guericke University, Magdeburg, Germany; ${ }^{\mathrm{d}}$ Department of Intensive Care, German Heart Center Leipzig, University Clinic, Leipzig, Germany; ${ }^{\mathrm{e}}$ Department of Intensive Care, The Austin Hospital, Melbourne, Australia; ${ }^{\mathrm{f}}$ Division of Cardiovascular Medicine, University of California, San Diego, La Jolla, Calif; ' ${ }^{\mathrm{g}}$ Department of Anesthesiology, The

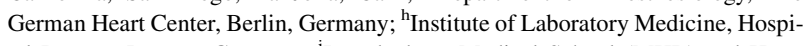

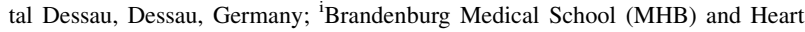
Center Brandenburg, Department of Cardiology, Bernau, Germany; and ${ }^{\mathrm{j} I n s t i t u t e}$ of Social Medicine and Health Economics, Otto-von-Guericke University, Magdeburg, Germany.

The database used in this study was funded by grants from the German Heart Foundation (Deutsche Stiftung für Herzforschung, Frankfurt am Main, Germany),
}

the Else Kröner-Fresenius-Stiftung (Bad Homburg, Germany), the Canadian Intensive Care Foundation (Edmonton, Canada), the Intensive Care Foundation (Melbourne, Australia), and the Austin Hospital ICU Research Fund (Melbourne, Australia).

Received for publication March 14, 2017; revisions received Nov 13, 2017; accepted for publication Dec 15, 2017; available ahead of print Feb 1, 2018.

Address for reprints: Anja Haase-Fielitz, PharmD, Institute of Social Medicine and Health Economics, Otto-von-Guericke University Magdeburg, Leipziger Straße 44, 39120 Magdeburg, Germany (E-mail: Anja.Haase-Fielitz@med.ovgu.de). $0022-5223 / \$ 36.00$

Copyright (C) 2017 by The American Association for Thoracic Surgery https://doi.org/10.1016/j.jtcvs.2017.12.056 


\section{Abbreviations and Acronyms \\ AKI = acute kidney injury \\ $\mathrm{CKD}=$ chronic kidney disease \\ $\mathrm{CPB}=$ cardiopulmonary bypass \\ $\mathrm{CRP}=\mathrm{C}$-reactive protein \\ IL-6 = interleukin 6 \\ KDIGO $=$ Kidney Disease: Improving Global Outcomes \\ MAKE $=$ major adverse kidney event \\ NGAL $=$ neutrophil gelatinase-associated lipocalin \\ RIFLE = renal risk, injury, failure, loss of renal function, end-stage renal disease classification \\ RRT = renal replacement therapy}

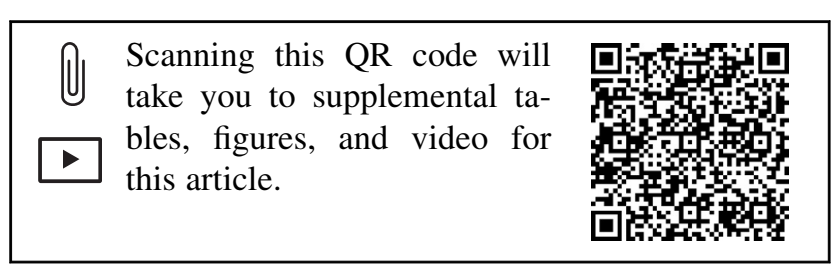

outcomes appeared to translate into long-term outcomes.ConclusionsUrinary kidney biomarkers identified RIFLEnegative patients with high-risk subclinical AKI as well as a higher risk subgroup of patients among RIFLE-AKIpositive patients. These findings support the concept that urinary biomarkers define subclinical AKI and higher risk subpopulations with worse long-term prognosis among standard patients with AKI. (J Thorac Cardiovasc Surg 2018;155:2441-52)

Acute kidney injury (AKI) is a common postoperative complication in critically ill patients after cardiac surgery. ${ }^{1-3}$ The consensus definition of AKI is currently based on changes in serum creatinine and urine output. However, recent data have challenged this paradigm. A pooled analysis of prospective cohort studies highlighted the prognostic relevance of combining routine kidney function parameters with kidney injury markers. ${ }^{4}$ Kidney biomarker test results identified patients with AKI being at increased risk of adverse outcomes. ${ }^{4}$ Also, a condition referred to as subclinical AKI (positive biomarker status without creatinine- or urine output-based criteria for AKI) was identified. ${ }^{5}$ On the basis of studies predominantly using neutrophil gelatinase-associated lipocalin (NGAL) for outcome prediction, ${ }^{4,6,7}$ kidney biomarkers have been suggested to complement serum creatinine- or urine output-based criteria for AKI diagnosis. ${ }^{8}$ Despite its obvious importance, this new concept has not been sufficiently validated. ${ }^{9}$

In this study we chose NGAL, interleukin 6 (IL-6), midkine, and total protein measured in urine as AKI biomarkers. ${ }^{10-16}$

A concern about all of these biomarkers relates to the fact that they may simply represent markers of inflammation rather than renal injury itself because they have not been adjusted for the degree of inflammation using nonrenal markers of postcardiac surgery inflammation such as C-reactive protein (CRP). ${ }^{17,18}$

Accordingly, the aim of this study was to evaluate the performance and degree of agreement of multiple different urinary kidney biomarkers according to AKI subtypes (ie, biomarker/RIFLE status [- or +$]$ ) in relation to shortand long-term outcomes of patients undergoing cardiac surgery and to control findings for a key marker of systemic inflammation (ie, CRP). Also, the frequency and type of subclinical AKI (positivity of any biomarker but RIFLE negativity) should be determined.

\section{SUBJECTS AND METHODS}

This ancillary study of the Bicarbonate-Multicenter Study used a cohort of 200 patients who underwent elective heart surgery with the use of cardiopulmonary bypass (CPB) enrolled at a study center, specifically the German Heart Center Berlin, (NCT00672334) from January 2009 through June 2010 (Figure 1). Full study details were described previously. ${ }^{19}$

\section{Patients}

In brief, we enrolled adult cardiac surgery patients at increased risk of AKI at a tertiary hospital (Table E1). ${ }^{19}$ We excluded patients with chronic renal impairment (preoperative serum creatinine $>300 \mu \mathrm{mol} / \mathrm{L}$ ), those undergoing an emergency cardiac surgery procedure, patients on immunosuppression therapy, and those enrolled in a conflicting research study. The institutional ethics committees granted permission to collect data; conduct biomarker measurement; and track long-term outcomes, including contact with patients and their physicians for this study (Charité University Medicine, Berlin, Germany No. ZS EK 11 654/07 and University of Magdeburg, Magdeburg, Germany No. 61/14;2014). Written informed consent from all patients was obtained.

\section{Biomarker Measurements}

Urine and blood samples were obtained preoperatively and at 6 and 24 hours after commencement of CPB, immediately centrifuged at $5000 \mathrm{rpm}$, and stored at $-80^{\circ} \mathrm{C}$. For measurement of urinary NGAL, the Architect platform (Abbott Diagnostics, Abbott Park, Ill) was used $(10-1500 \mathrm{ng} / \mathrm{mL}$, imprecision $\leq 10 \%)$. Urinary IL-6 and serum CRP were determined using Cobas e/c411 Immunoassay Analyzer Platform (ROCHE Diagnostics, Mannheim, Germany) (IL-6, 1.5-5.000 pg/mL; variability $<9 \%$; CRP, $0.3-350 \mathrm{mg} / \mathrm{L}$; variability $<5 \%$ ). Urinary midkine was measured using human midkine sandwich enzyme-linked immunosorbant assay development kit (PeproTech, Hamburg, Germany) $(15-2000 \mathrm{pg} / \mathrm{mL}$ analyzed by spectrophotometer TECAN Infinite 200; Tecan Group Inc, Durham, NC). Urine dipstick for assessment of proteinuria (Boehringer Ingelheim Pharma, Ingelheim am Rhein, Germany) was performed at all study measurement time points and 


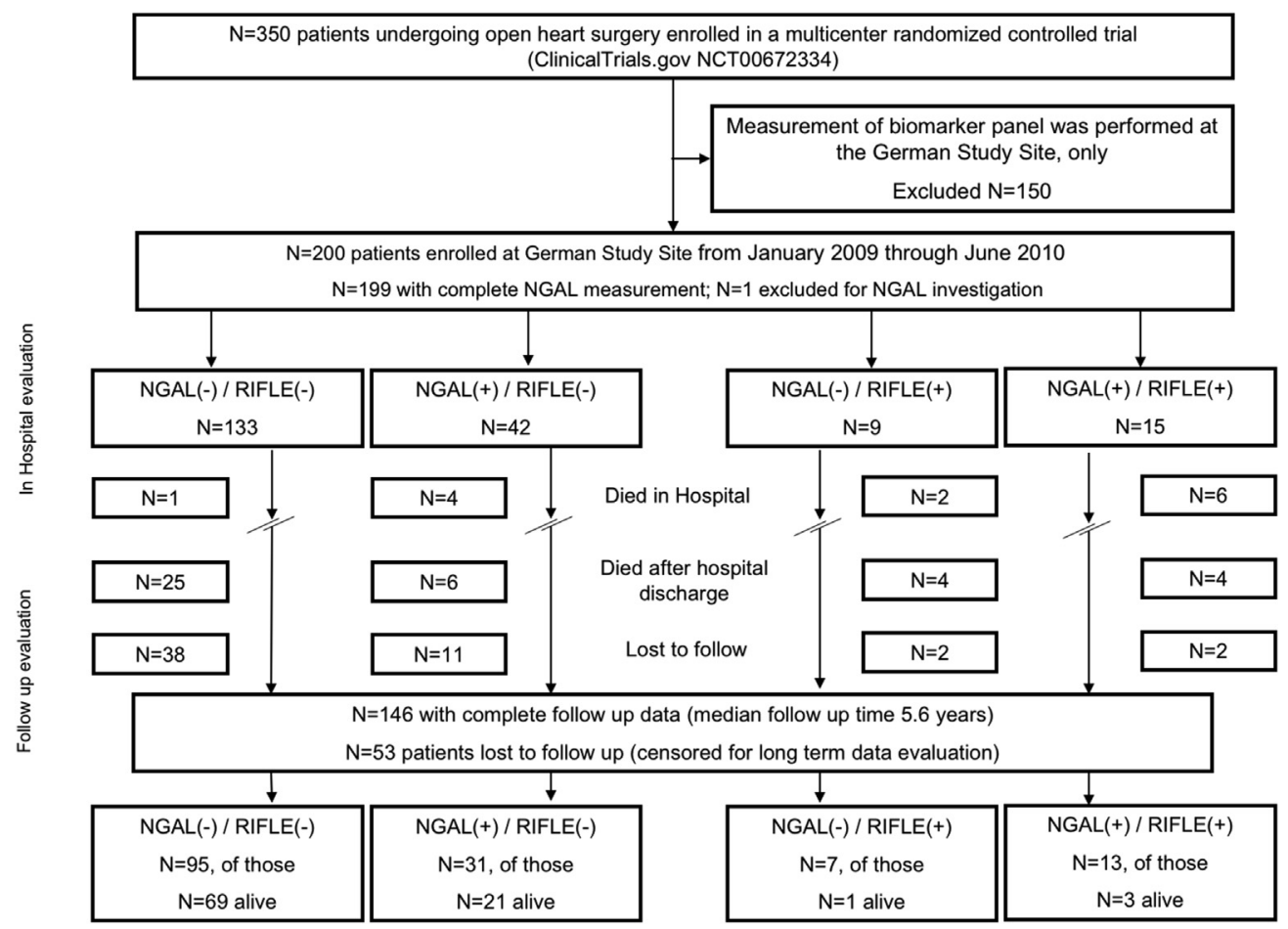

FIGURE 1. Patient flow chart through the study (exemplary for neutrophil gelatinase associated lipocalin/risk injury failure end-stage renal disease classification $[N G A L / R I F L E]$ status).

quantified to the manufacturer's instructions using the dipstick's scale (ie, none,,+++ , or +++ ).

Biomarker test results were available in 199 out of 200 patients for NGAL, 195 patients for midkine, 190 patients for IL-6, 199 patients for proteinuria, and 194 patients for CRP.

\section{AKI and Chronic Kidney Disease Definition}

AKI was defined according to the renal risk, injury, failure, loss of renal function, end-stage renal disease classification (RIFLE) criteria using increases of postoperative serum creatinine level from preoperative baseline and decline in urine output, both assessed during the first 7 postoperative days. ${ }^{20}$ We used RIFLE criteria to enable comparability with previous studies ${ }^{4,6,7}$ and because literature indicates a trend for higher discriminative value in predicting hospital mortality in cardiac surgery patients. ${ }^{21,22}$ Chronic kidney disease (CKD) stages were reported according to the Kidney Disease: Improving Global Outcomes (KDIGO) guidelines. $^{23}$

\section{Patient Allocation According to AKI Subtypes}

For allocation of patients according to AKI subtypes, RIFLE and biomarker status was determined for each patient. In our patient cohort, urinary kidney biomarker positivity $(+)$ or biomarker negativity $(-)$ was defined as a biomarker concentration measured at 6 hours after commencement of $\mathrm{CPB}$ above or below a biomarker-specific cut-off value, as determined by the maximum Youden's index (ie, optimum cut-off point for combined sensitivity and specificity for reported end points). ${ }^{24}$ The following cut-off values for AKI were determined: NGAL $\geq 50 \mathrm{ng} / \mathrm{mL}$, midkine $\geq 250 \mathrm{pg} / \mathrm{mL}$, IL- $6 \geq 11 \mathrm{pg} / \mathrm{mL}$, proteinuria $\geq 30 \mathrm{mg} / \mathrm{dL}$ (dipstick-equivalent), CRP $\geq 3 \mathrm{mg} / \mathrm{L}$, and CRP at 24 hours $\geq 67 \mathrm{mg} / \mathrm{L}$. Area under the curve-receiver operating characteristics values and receiver operating characteristics comparisons are provided in Tables E1 through E17 and Figures E1 and E2.

We used NGAL/midkine/IL-6/proteinuria (+) or NGAL/midkine/IL-6/ proteinuria (-) to indicate the presence or absence of biomarker-positive AKI. ${ }^{4}$ For CRP, the terms were adapted accordingly. Proteinuria positivity was defined as early postoperative de novo or worsening proteinuria (according to change in semiquantitative dipstick scale) compared with preoperative value avoiding carryover effects of preoperative CKD.

In summary, 4 different combinations could be distinguished considering RIFLE status and urinary biomarker status:

$$
\begin{aligned}
& \text { Biomarker (-)/RIFLE }(-), \text { Biomarker }(+) / \operatorname{RIFLE}(-), \\
& \text { Biomarker }(-) / \operatorname{RIFLE}(+), \text { Biomarker }(+) / \operatorname{RIFLE}(+) .
\end{aligned}
$$

To calculate the proportion of patients with AKI additionally identified by urinary kidney biomarkers only and not by RIFLE criteria (subclinical AKI) in relation to the proportion of patients diagnosed to have AKI by conventional, RIFLE-based criteria, we used the formula

$$
\begin{gathered}
\text { Biomarker }(+) / \boldsymbol{R I F L E}(-) \\
=\frac{\text { Benal impairment identified by urinary biomarker }}{\text { all renal impairment }}
\end{gathered}
$$




\section{Patient Follow-up}

We obtained patient kidney and vital status at 90 days postoperative as well as at least 5 years after discharge through various mechanisms and cross-referenced when possible. We performed telephone calls and contact by mail to patients' homes and physicians and reviewed hospital and physicians' records. Serum creatinine; urine protein/albumin concentrations, if available; death status; and date of death were recorded. As of July 2015, data collection was closed.

\section{Study End Points}

For the purpose of this study on AKI subtypes, patient-relevant end points were predefined before conduction of statistical analysis. The primary end point was a combination of acute renal replacement therapy (RRT) or in-hospital mortality. Secondary end point, major adverse kidney events (MAKE) was defined as the occurrence of 1 or more of the following criteria: sustained AKI (stages I or F without recovery within 3 days), acute RRT, or in-hospital mortality. Long-term outcomes consisted of long-term mortality and development of worsening of preoperatively present CKD (Figure E1) according to KDIGO stages.

\section{Statistical Analysis}

Clinicians caring for the patients remained blinded to biomarker test results and laboratory investigators remained blinded to patient outcomes. Analysis of variance, Mann-Whitney $U$ test, Kruskal-Wallis test, Pearson $\chi^{2}$ test, or Fisher exact test were used where appropriate. The odds ratio (OR) and 95\% confidence interval (CI) were calculated for assessment of risk disparity between biomarker/RIFLE groups. We performed measurement of agreement among all biomarkers testing for $\kappa$ variables and interpreted results according to Landis and Koch (Table E17). ${ }^{25}$

Multivariate regression analyses were undertaken to assess robustness of urinary biomarkers as independent predictors of RRT or in-hospital mortality based on a previously published reference model, ${ }^{26}$ including a urinary biomarker as well as chronic heart failure, preoperative estimated glomerular filtration rate, peripheral vascular disease, sex, chronic obstructive pulmonary disease, type of surgery, and repeated cardiac surgery as independent variables.

Cox proportional-hazard regression models adjusted for baseline group differences were performed for confirmation of primary and secondary end points (age, type of surgery [eg, coronary artery bypass graft vs concomitant surgery], previous cardiac surgery, and perioperative fluid balance at 0-6 hours).

Influences of cardiovascular risk factors, baseline renal function, and biomarker/RIFLE group allocation on long-term patient survival were assessed using cox proportional-hazard regression models. Kaplan-Meier curves are presented. Differences of curves were evaluated using log-rank test, Breslow test, and Tarone-Ware test. SPSS for Windows, version 22.0 (IBM-SPSS Inc, Armonk, NY) and MedCalc version 17.6 (MedCalc Software, Ostend, Belgium) were used for statistical analysis.

\section{RESULTS}

\section{Pre- and Perioperative Characteristics of Patients}

Patient characteristics according to NGAL/RIFLE-status are presented in Tables 1 and 2. For midkine/IL-6/ proteinuria/CRP and RIFLE status, patient characteristics are

TABLE 1. Preoperative patient characteristics according to urine neutrophil gelatinase-associated lipocalin/risk injury failure end-stage renal disease classification (NGAL/RIFLE) subgroup $^{20}$

\begin{tabular}{|c|c|c|c|c|c|}
\hline Variable & $\frac{\text { NGAL }(-) / \text { RIFLE }(-)}{(n=133 ; 66.8 \%)}$ & $\frac{\text { NGAL }(+) / \operatorname{RIFLE}(-)}{(\mathrm{n}=42 ; 21.1 \%)}$ & $\frac{\text { NGAL }(-) / \text { RIFLE }(+)}{(\mathrm{n}=9 ; 4.5 \%)}$ & $\frac{\text { NGAL }(+) / \text { RIFLE }(+)}{(n=15 ; 7.5 \%)}$ & $\begin{array}{c}P \\
\text { value }\end{array}$ \\
\hline Age (y) & $68(58-73)$ & $70.5(62.8-76.3)$ & $73(69-77)$ & $72(67-77)$ & .033 \\
\hline Female sex & $40(30.1)$ & $11(26.2)$ & $2(22.2)$ & $6(40.0)$ & .763 \\
\hline Body mass index & $26.5(23.4-29.4)$ & $26.1(24.5-28.4)$ & $28(25.6-30.6)$ & $26.1(24.6-31.6)$ & .181 \\
\hline Insulin dependent diabetes mellitus & $7(5.3)$ & $2(4.8)$ & $0(0)$ & $0(0)$ & 1.000 \\
\hline Non-insulin dependent diabetes mellitus & $25(18.8)$ & $9(21.4)$ & $0(0)$ & $5(33.3)$ & .261 \\
\hline Arterial hypertension & $97(72.9)$ & $33(78.6)$ & $9(100)$ & $14(93.3)$ & .109 \\
\hline Hypercholesterolemia & $83(62.4)$ & $30(71.4)$ & $7(77.8)$ & $10(66.7)$ & .611 \\
\hline Chronic obstructive pulmonary disease & $21(15.8)$ & $6(14.3)$ & $2(22.2)$ & $3(20.0)$ & .844 \\
\hline Smoking habit, current & $23(17.3)$ & $4(9.5)$ & $3(33.3)$ & $1(6.7)$ & .227 \\
\hline Preoperative serum creatinine $(\mu \mathrm{mol} / \mathrm{L})$ & $86.6(76.0-106.1)$ & $97.2(80.4-114.9)$ & $97.2(86.6-123.8)$ & $97.2(73.4-132.6)$ & .092 \\
\hline Peripheral vascular disease & $36(27.1)$ & $8(19)$ & $4(44.4)$ & $4(26.7)$ & .414 \\
\hline Left ventricular dysfunction* & $28(21.1)$ & $6(14.3)$ & $3(33.3)$ & $4(26.7)$ & .454 \\
\hline Left ventricular ejection fraction $(\%)$ & $55.0(40.0-60.0)$ & $50(43.8-60)$ & $45(30-60)$ & $55(50-60)$ & .436 \\
\hline \multicolumn{6}{|l|}{ Preoperative medication } \\
\hline ACE inhibitor/AT-II antagonist & $68(51.1)$ & $28(66.7)$ & $6(66.7)$ & $12(80)$ & .070 \\
\hline Beta-blocker & $96(72.2)$ & $32(76.2)$ & $7(77.8)$ & $11(73.3)$ & .978 \\
\hline Calcium channel blocker & $39(29.3)$ & $10(23.8)$ & $4(44.4)$ & $6(40)$ & .444 \\
\hline HMG-CoA reductase inhibitor & $77(57.9)$ & $24(57.1)$ & $6(66.7)$ & $9(60)$ & .958 \\
\hline Diuretics & $81(60.9)$ & $27(64.3)$ & $6(66.7)$ & $14(93.3)$ & .102 \\
\hline
\end{tabular}

Values are presented as median (25th-75th percentile) or frequency (\%). NGAL, Neutrophil gelatinase-associated lipocalin; RIFLE, renal risk, injury, failure, loss of renal function, end-stage renal disease classification; $A C E$, angiotensin-converting enzyme; AT-II, angiotensin-II; HMG-CoA, 3-hydroxy-3-methyl-glutaryl-coenzyme A. *Left ventricular ejection fraction $<35 \%$. 
TABLE 2. Perioperative patient characteristics according to neutrophil gelatinase-associated lipocalin/risk injury failure end-stage renal disease classification (NGAL/RIFLE) $\operatorname{subgroup}^{20}$

\begin{tabular}{|c|c|c|c|c|c|}
\hline Variable & $\frac{\text { NGAL }(-) / \operatorname{RIFLE}(-)}{(\mathrm{n}=133 ; 66.8 \%)}$ & $\frac{\operatorname{NGAL}(+) / \text { RIFLE }(-)}{(\mathrm{n}=42 ; 21.1 \%)}$ & $\frac{\text { NGAL }(-) / \operatorname{RIFLE}(+)}{(\mathrm{n}=9 ; \mathbf{4 . 5} \%)}$ & $\frac{\operatorname{NGAL}(+) / \operatorname{RIFLE}(+)}{(\mathrm{n}=15 ; \mathbf{7 . 5} \%)}$ & $\begin{array}{c}P \\
\text { value }\end{array}$ \\
\hline \multicolumn{6}{|l|}{ Procedures } \\
\hline CABG surgery & $29(21.8)$ & $3(7.1)$ & $3(33.3)$ & $1(6.7)$ & .048 \\
\hline Valvular surgery & $64(48.1)$ & $13(31.0)$ & $2(22.2)$ & $10(66.7)$ & .037 \\
\hline CABG and valvular surgery & $28(21.1)$ & $20(47.6)$ & $3(33.3)$ & $3(20)$ & .008 \\
\hline Thoracic aortic surgery & $11(8.3)$ & $6(14.3)$ & $0(0)$ & $1(6.7)$ & .575 \\
\hline Ventricular assist device & $1(0.8)$ & $0(0)$ & $1(11.1)$ & $0(0)$ & .170 \\
\hline Redo cardiac surgery & $26(19.5)$ & $16(38.1)$ & $3(33.3)$ & $7(46.7)$ & .016 \\
\hline $\begin{array}{l}\text { Duration of cardiopulmonary } \\
\text { bypass (min) }\end{array}$ & $115(90-140)$ & $140(109-190)$ & $157(87-189)$ & $209(129-368)$ & $<.001$ \\
\hline $\begin{array}{l}\text { Lowest intraoperative MAP } \\
\quad(\mathrm{mm} \mathrm{Hg})\end{array}$ & $35(30-41)$ & $34(28.8-38)$ & $31(22-36.5)$ & $31(24-41)$ & .209 \\
\hline \multicolumn{6}{|l|}{$\begin{array}{l}\text { Perioperative fluid balance and } \\
\text { medication }(0-24 \mathrm{~h})\end{array}$} \\
\hline Fluid intake (mL) & $8512(7012-9764)$ & $7162(5346-9213)$ & $6356(5506-7565)$ & $5856(3724-7712)$ & $<.001$ \\
\hline Urine output (mL) & $5490(4502-6525)$ & $5227(4097-5796)$ & $4020(2795-4277)$ & $3850(3125-4490)$ & $<.001$ \\
\hline Packed red blood cells (mL) & $250(0-500)$ & $500(187-1000)$ & $500(0-1625)$ & $1000(500-2500)$ & $<.001$ \\
\hline Drain output $(\mathrm{mL})$ & $500(350-695)$ & $500(368-841)$ & $525(299-1550)$ & $950(700-2125)$ & .001 \\
\hline Fluid balance $(\mathrm{mL})$ & $2952(1847-4199)$ & $1872(556-3271)$ & $2972(2296-3889)$ & $1696(-99$ to 4756$)$ & $<.001$ \\
\hline Furosemide (mg) & $20(0-40)$ & $40(20-52)$ & $60(20-110)$ & $80(20-272)$ & $<.001$ \\
\hline \multicolumn{6}{|l|}{ Urine NGAL $(\mathrm{ng} / \mathrm{mL})$} \\
\hline $0 \mathrm{~h}$ & $2.1(0-6.1)$ & $2.65(0-5.25)$ & $5.3(0-7.1)$ & $7.4(2.6-36.7)$ & .064 \\
\hline $6 \mathrm{~h}$ & $4.4(0-12.4)$ & $201.7(86.6-410.7)$ & $6.9(2.3-15.5)$ & $292.3(142.3-570.4)$ & $<.001$ \\
\hline $24 \mathrm{~h}$ & $5.2(0-11.8)$ & $7.7(1.8-34.9)$ & $12(2.9-15.8)$ & $52.9(19.8-119.8)$ & $<.001$ \\
\hline RIFLE stages & & & & & .381 \\
\hline Risk & & & $6(66.7)$ & $7(46.7)$ & \\
\hline Injury & & & $3(33.3)$ & $4(26.7)$ & \\
\hline Failure & & & $0(0)$ & $4(26.7)$ & \\
\hline
\end{tabular}

Values are presented as median (25th-75th percentile) or frequency (\%). NGAL, Neutrophil gelatinase-associated lipocalin; RIFLE, renal risk, injury, failure, loss of renal function, end-stage renal disease classification; $C A B G$, coronary artery bypass graft; $M A P$, mean arterial pressure.

shown in Tables E3-E9. Patient flow through study is illustrated in Figure 1.

All urinary biomarkers but not CRP were found to be independent predictors of the primary end point RRT or in-hospital mortality in a multivariate clinical model based on the Cleveland risk assessment model provided in Table 3 . $^{26}$

The majority of patients $(66.8 \%)$ was classified as NGAL(-)/RIFLE(-) (ie, no renal injury), $21.1 \%$ as NGAL $(+) / \operatorname{RIFLE}(-)$ (ie, subclinical AKI), $4.5 \%$ as NGAL $(-) / \operatorname{RIFLE}(+)$ (ie, isolated functional AKI), and $7.5 \%$ as $\mathrm{NGAL}(+) / \mathrm{RIFLE}(+)$ (ie, higher risk functional AKI) (Tables 1 and 2).

Within all 4 NGAL/RIFLE groups, patients were virtually similar with regard to demographic data, comorbidities, and preoperative medications except for age. $\operatorname{RIFLE}(+)$ patients were older than $\operatorname{RIFLE}(-)$ patients independent of NGAL status. More patients with NGAL positivity received operations other than coronary artery bypass grafting. Significant group differences existed for duration of CPB, fluid intake/balance, drain output, urine output, and transfusion of packed red blood cells and furosemide (all $P$ values $<.001$ ).

\section{AKI Subgroups: RIFLE-AKI, Subclinical AKI, and Single-Biomarker-Positive Subclinical AKI}

In our study cohort, $24(12 \%)$ patients developed RIFLE-positive AKI.

NGAL positivity in RIFLE-negative patients additionally identified 42 patients with suspected subclinical AKI $(+63.6 \%)$ compared with RIFLE-based criteria only. Moreover, using midkine, 33 patients $(+57.9 \%)$, or IL-6, 58 patients $(+74.4 \%)$, were additionally identified as having subclinical AKI. Finally, using dipstick proteinuria, 95 patients $(+79.8 \%)$ were additionally identified.

Eight out of 200 patients $(4 \%)$ were only NGAL $(+) /$ RIFLE $(-)$ but not midkine $(+)$ or IL-6(+). Six patients $(3 \%)$ were midkine $(+) / \operatorname{RIFLE}(-)$ but not $\mathrm{NGAL}(+)$ or IL-6(+). Twenty-two patients $(11 \%)$ were only IL-6(+)/ RIFLE(-) but not NGAL(+) or midkine(+). 
TABLE 3. Multivariate logistic regression analysis of risk factors for the prediction of renal replacement therapy (RRT) or in-hospital mortality based on the Cleveland risk assessment model*

\begin{tabular}{|c|c|c|c|c|c|c|c|c|c|c|}
\hline & \multicolumn{2}{|l|}{ Model 0} & \multicolumn{2}{|l|}{ Model 1} & \multicolumn{2}{|l|}{ Model 2} & \multicolumn{2}{|l|}{ Model 3} & \multicolumn{2}{|l|}{ Model 4} \\
\hline & $\operatorname{Exp}(B)$ & $P$ & $\operatorname{Exp}(\mathbf{B})$ & $P$ & $\operatorname{Exp}(\mathbf{B})$ & $P$ & $\operatorname{Exp}(\mathbf{B})$ & $P$ & $\operatorname{Exp}(B)$ & $P$ \\
\hline & $(95 \%$ CI $)$ & & $(95 \%$ CI $)$ & value & $(95 \%$ CI $)$ & value & $(95 \%$ CI $)$ & value & $(95 \%$ CI $)$ & value \\
\hline CHF & $1.264(0.397-4.027)$ & .692 & $1.556(0.454-5.331)$ & .482 & $1.262(0.363-4.391)$ & .714 & $1.849(0.630-5.427)$ & .263 & $1.414(0.405-4.945)$ & .587 \\
\hline $\begin{array}{c}\text { Preoperative } \\
\text { eGFR }\end{array}$ & $0.970(0.946-0.995)$ & .020 & $0.967(0.940-0.994)$ & .017 & $0.963(0.937-0.991)$ & .009 & $0.969(0.942-0.996)$ & .024 & $0.964(0.128-1.899)$ & .009 \\
\hline PVD & $0.418(0.114-1.530)$ & .187 & $0.554(0.144-2.130)$ & .390 & $0.553(0.144-2.117)$ & .387 & $2.141(0.772-5.936)$ & .772 & $0.494(0.128-1.899)$ & .305 \\
\hline COPD & $1.639(0.535-5.022)$ & .387 & $1.855(0.572-6.018)$ & .304 & $2.152(0.677-6.843)$ & .194 & $1.672(0.515-5.430)$ & .392 & $1.583(0.487-5.144)$ & .445 \\
\hline Sex & $0.394(0.145-1.069)$ & .067 & $0.290(0.098-0.857)$ & .025 & $0.484(0.166-1.410)$ & .183 & $1.816(0.547-6.035)$ & .330 & $0.471(0.158-1.404)$ & .177 \\
\hline $\begin{array}{l}\text { Type of } \\
\text { surgery }\end{array}$ & $0.738(0.246-2.216)$ & .588 & $0.547(0.169-1.767)$ & .313 & $0.731(0.236-2.266)$ & .587 & $0.715(0.218-2.342)$ & .580 & $0.442(0.125-1.560)$ & .204 \\
\hline $\begin{array}{l}\text { Previous cardiac } \\
\text { surgery }\end{array}$ & $1.325(0.421-4.169)$ & .630 & $2.359(0.628-8.859)$ & .203 & $1.321(0.402-4.341)$ & .646 & $0.498(0.168-1.475)$ & .208 & $2.001(0.537-7.459)$ & .302 \\
\hline $\begin{array}{l}\text { Urinary NGAL } \\
\qquad(\mathrm{ng} / \mathrm{mL})\end{array}$ & & & $1.003(1.001-1.005)$ & $<.001$ & & & & & & \\
\hline $\begin{array}{l}\text { Urinary midkine } \\
\qquad(\mathrm{pg} / \mathrm{mL})\end{array}$ & & & & & $1.001(1.000-1.001)$ & .001 & & & & \\
\hline $\begin{array}{l}\text { Urinary IL-6 } \\
\text { (pg/mL) }\end{array}$ & & & & & & & $1.001(1.000-1.001)$ & .05 & & \\
\hline $\begin{array}{c}\text { Plasma CRP } \\
(\mathrm{mg} / \mathrm{L})\end{array}$ & & & & & & & & & $0.992(0.957-10.28)$ & .652 \\
\hline $\begin{array}{l}95 \% C I, 95 \% \text { Con } \\
\text { pulmonary disease; } \\
1-4 \text { ) into multivariat } \\
\text { prediction of the prin } \\
\text { obstructive pulmona } \\
\text { mortality. Goodness } \\
R^{2}=0.148 ; \text { model } 1 \\
\text { Nagelkerke } R^{2}=0 . \\
\text { likelihood }=112.53\end{array}$ & $\begin{array}{l}\text { ate regression analysis t } \\
\text { imary end point in-hospit } \\
\text { s of fit of the model impre } \\
1 \text { Hosmer-Lemeshow } P \text {. } \\
0.256 \text {; model } 3 \text { Hosmer- } \\
3 \text {, Nagelkerke } R^{2}=0.16\end{array}$ & $\begin{array}{l}\text { gery, pr } \\
\text { roved c } \\
.127 \text { an } \\
\text {-Lemes } \\
65 .\end{array}$ & $\begin{array}{l}\text { ude interaction. Multiva } \\
\text { tality or RRT. Chronic h } \\
\text { evious cardiac surgery. } \\
\text { onsiderably after adding } \\
\text { ad }-2 \log \text { likelihood }= \\
\text { how } P .272 \text { and }-2 \log \end{array}$ & $\begin{array}{l}\text { All asse } \\
\text { urinary } \\
104.58, \\
\text { likelih }\end{array}$ & $\begin{array}{l}\text { gistic regression analy } \\
\text { ure, preoperative estim } \\
\text { essed urinary biomarke } \\
\text { biomarkers: model } 0 \mathrm{H} \\
\text { Nagelkerke } R^{2}=0.282 \\
\text { ood }=91.90, \text { Nagelker }\end{array}$ & $\begin{array}{l}\text { ros could } \\
\text { tosmer- } \\
\text {; model } \\
\text { rke } R^{2}=\end{array}$ & $\begin{array}{l}\text { ded variables based on } \\
\text { merular filtration rate, } \\
\text { be identified as indepe } \\
\text { Lemeshow } P .629 \text { and }- \\
2 \text { Hosmer-Lemeshow } P \\
=0.286 \text {; and model } 4 \text { H }\end{array}$ & P.752a & $\begin{array}{l}\text { arker one atter anothe } \\
\text { leveland reference mo } \\
\text { al vascular disease, sex } \\
\text { redictors of RRT or in } \\
\text { kelihood }=119.30, \mathrm{~N} \\
\text { nd }-2 \text { log likelihood } \\
\text {-Lemeshow } P .308 \text { an }\end{array}$ & $\begin{array}{l}\text { (model } \\
\text { le }{ }^{26} \text { for } \\
\text { chronic } \\
\text { hospital } \\
\text { elkerke } \\
106.84 \text {, } \\
-2 \log \end{array}$ \\
\hline
\end{tabular}

\section{Patient Outcomes According to Urinary Kidney Biomarker/RIFLE Status}

In multivariable Cox regression analyses adjusted for clinically relevant baseline differences, allocation to all biomarker/RIFLE groups was independently associated with the primary and secondary end points (Tables E13-E15).

Independent of RIFLE status, urinary kidney biomarkerpositive patients had worse outcomes compared with urinary biomarker-negative patients. Worst outcomes were found in $\mathrm{NGAL}(+)$ or midkine $(+)$ or $\mathrm{IL}-6(+)$ or proteinuria( $(+)$ and $\operatorname{RIFLE}(+)$ patients, whereas best outcomes in biomarker(-) and RIFLE $(-)$ patients (see Figure 2 and Table E16).

The outcome patterns for initiation of acute RRT, in-hospital mortality, combined RRT or in-hospital mortality, and for MAKE were similar with urinary NGAL, midkine, and IL-6 (Figure 2), but less pronounced with de novo or worsening proteinuria (Figure 2,D) and not detected by CRP (Figure 3).

Primary end point. ORs for the primary end point (acute RRT or in-hospital mortality), in NGAL(+)/RIFLE(-) or in IL-6(+)/RIFLE $(-)$ patients (subclinical AKI) versus
NGAL(-)/RIFLE(-) or IL-6(-)/RIFLE(-) patients was $5.86(95 \% \mathrm{CI}, 1.34-25.65[P=.020]$ and adjusted hazard ratio (HR)), $7.18(95 \% \mathrm{CI}, 1.52-33.93[P=.013])$ (Table 4) for NGAL and $6.35(95 \% \mathrm{CI}, 1.24-32.52$ $[P=.020]$ and adjusted $\mathrm{OR}, 6.27 ; 95 \% \mathrm{CI}, 1.12-35.21$ $[P=.037]$ ), for IL-6, respectively (Figure $2, A$ and $B$, and Table 4).

ORs for the primary end point, in NGAL $(+) / \operatorname{RIFLE}(+)$ or midkine $(+) / \operatorname{RIFLE}(+)$ versus $\operatorname{NGAL}(-) / \operatorname{RIFLE}(+)$ or midkine $(-) / \operatorname{RIFLE}(+)$ patients were $9.6(95 \% \mathrm{CI}$, 1.4-67.3; $P=.033)$ and $14.7(95 \%$ CI, 2.0-109.2; $P=.011)$, respectively. After adjustment for baseline differences all biomarkers showed a stepwise increase in adjusted HR for development of the primary end point. Midkine and proteinuria detected only nonsignificantly different risk profiles for subclinical AKI versus biomarker(-)/RIFLE(-) patients (Table 4 and Table E15).

Biomarker NGAL/midkine/IL-6/proteinuria-positive/ RIFLE-positive status indicated increased risk for developing the primary end point compared with biomarker(-)/RIFLE(-) status with adjusted HRs ranging from 66 (for de novo or worsening proteinuria) to 100 (for NGAL) (all $P$ values $<.001$ [Table 4 and Table E16]). 

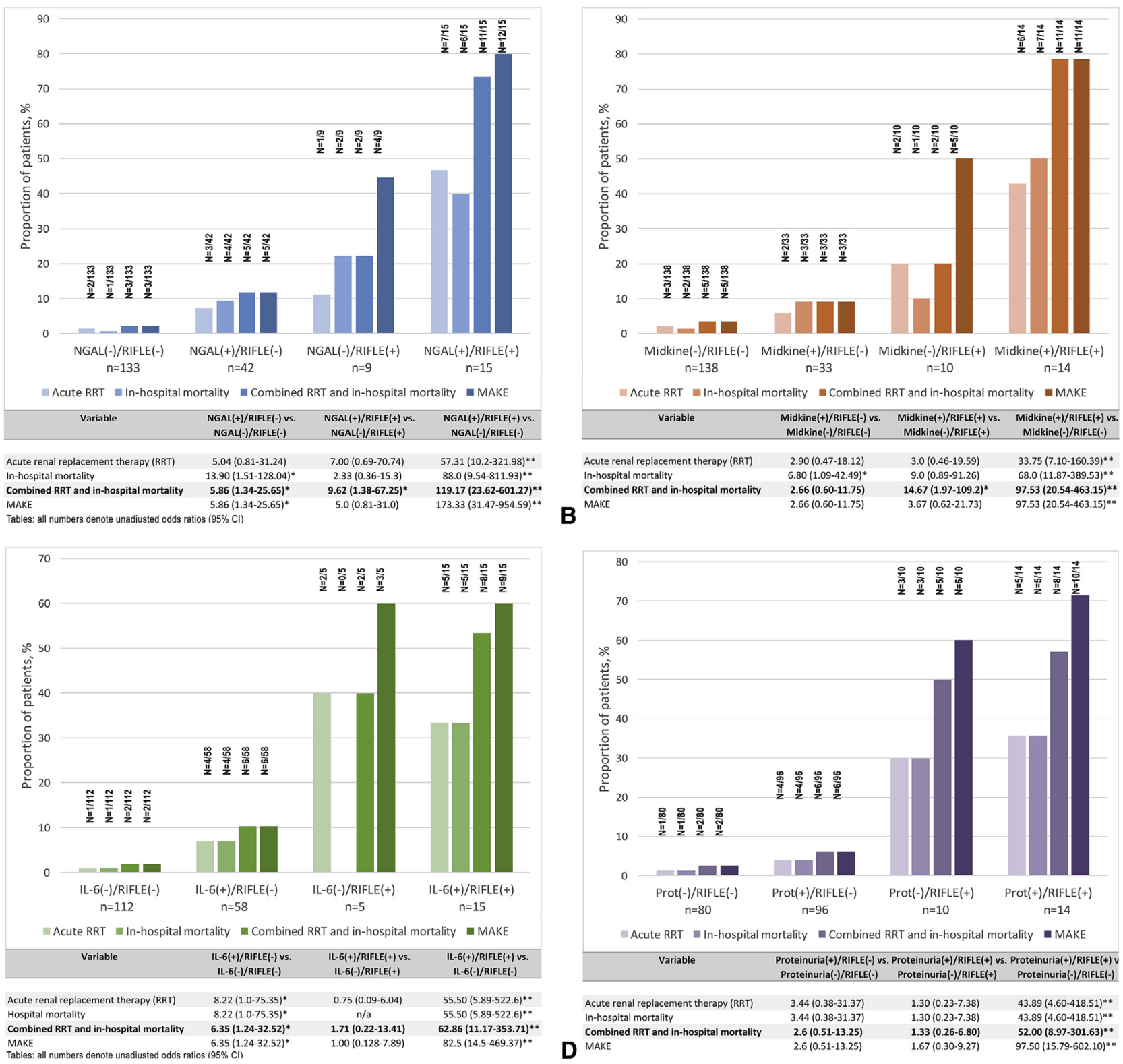

FIGURE 2. Proportion of patients with defined end points according to A, Neutrophil gelatinase-associated lipocalin $(N G A L)$ status. B, Midkine status. C, Interleukin-6 (IL-6) status. D, Proteinuria status and risk injury failure end-stage renal disease classification ${ }^{20}(R I F L E)$ status. The primary study end point, combination of acute renal replacement therapy $(R R T)$ or in-hospital mortality, is highlighted. Urinary biomarkers show a stepwise increase in all evaluated end points with biomarker positivity irrespective of RIFLE status. Underlying table shows unadjusted odds ratios and $95 \%$ confidence interval $(C I)$ for risk assessment between groups. Significance level for Pearson $\chi^{2}$ or Fisher exact test where appropriate. $* P<.05$. $* * P<.001 . M A K E$, Major adverse kidney events including in-hospital mortality, RRT initiation, and renal recovery from acute kidney injury.

Secondary end points. NGAL $(+) / \operatorname{RIFLE}(-)$ versus NGAL $(-) / \operatorname{RIFLE}(-)$ and $\operatorname{IL}-6(+) / \operatorname{RIFLE}(-)$ versus IL-6(-)/RIFLE(-) status had significantly increased risk of acute RRT, in-hospital mortality, or MAKE (ORs, 5.0-13.9; all $P$ values $<.05$ ) (Figure 2, $A$ and $C$ ). Occurrence of MAKE increased from $2.2 \%$ in $\operatorname{NGAL}(-) / \operatorname{RIFLE}(-)$ patients to $10.2 \%$ in $\operatorname{NGAL}(+) / \operatorname{RIFLE}(-)$ patients $(\mathrm{OR}$, 5.86; 95\% CI, 1.34-25.65; $P=.020$ ) (Figure $1, A$ ) and from $2.4 \%$ in IL-6(-)/RIFLE( $(-)$ to $11.0 \%$ in IL- $6(+) /$ RIFLE(+) patients (OR, 6.35; 95\% CI, 1.24-32.52; $P=.020$ ) (Figure $2, C$ ). For midkine $(+) / \operatorname{RIFLE}(-)$ versus midkine(-)/RIFLE(-) patients, a similar pattern for MAKE could be observed (OR, 2.66; $95 \% \mathrm{CI}$, 0.60-11.75) (Figure 2, B).

Patients with NGAL $(+) / \operatorname{RIFLE}(-)$ status had more than 10-times increased in-hospital mortality rate over patients 

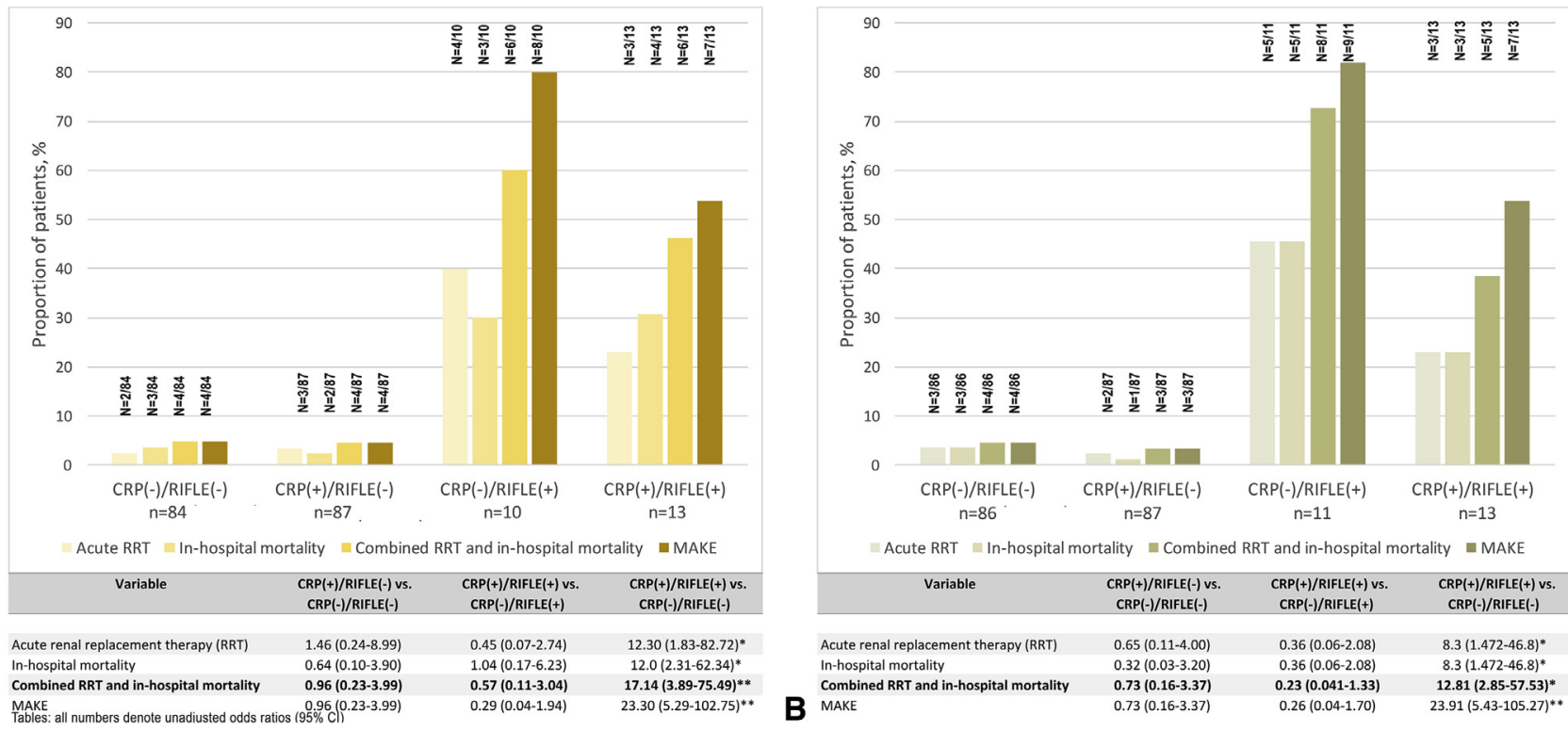

FIGURE 3. Proportion of patients with defined end points according to (A) C-reactive protein $(C R P)$ status at 6 hours. B, CRP status at 24 hours after commencement of cardiopulmonary bypass and risk injury failure end-stage renal disease classification ${ }^{20}(R I F L E)$ status. The primary study end point, combination of acute renal replacement therapy $(R R T)$ or in-hospital mortality, is highlighted. As opposed to urinary kidney biomarkers, for CRP (at 6 hours) no additive prognostic information was derived beyond RIFLE status. MAKE, Major adverse kidney events including in-hospital mortality, RRT initiation, and renal recovery from acute kidney injury.

without positive biomarker attribute $(0.8 \%$ in $\operatorname{NGAL}(-) /$ RIFLE $(-)$ group) versus $9.5 \%$ in the $\operatorname{NGAL}(+) / \operatorname{RILFE}(-)$ group (OR, 13.90; 95\% CI, 1.51-128.04; $P=.01$; adjusted HR, 13.02; 95\% CI, 1.34-126.96; $P=.027$ ) (Table 4).

Again, a similar pattern with similar magnitude was observed for midkine (OR for in-hospital mortality 6.80; 95\% CI, 1.09-42.49; $P=.05$ ) and IL-6 (OR, 8.22; 95\% CI, 1.0-75.35; $P<.05$ ) (Figure $2, A$ and $B$ ).

NGAL $(+) / \operatorname{RIFLE}(+)$ versus NGAL $(-) / \operatorname{RIFLE}(+)$ and midkine $(+) / \operatorname{RIFLE}(+)$ versus midkine(-)/RIFLE(-) status had nonsignificant increases in the risk of acute RRT, in-hospital mortality, or MAKE with OR ranging from 5.04 to 13.9 (Figure 2, $A$ and $B$ ).

Biomarker NGAL/midkine/IL-6/proteinuria(+)/RIFLE (+) status indicated increased risk of acute RRT, inhospital mortality, and MAKE compared with biomarker(-)/RIFLE(-) status with OR ranging from 44 (for acute RRT indicated by de novo or worsening proteinuria) to 173 (for MAKE indicated by NGAL) (all $P$ values $<.001$ ) (Figure 2, $A-D$ ).

\section{Long-Term Outcomes}

Median follow-up time was 5.6 years. Data from 147 $(73.5 \%)$ patients were collected at the final visit (52 patients $[35.4 \%]$ had died during follow-up). Fifty-three patients $(26.5 \%)$ were lost to follow-up (Figure 1).

Long-term follow-up is illustrated in Kaplan-Meier Curves (Figure 4, A) showing distinct separation between the biomarker and RIFLE groups. Separation for long-term survival comparing biomarker $(+)$ with biomarker(-) patients was found independent of RIFLE status (Figure 4, A). Survival curves of AKI subtypes separated early after cardiac surgery and continued during long-term follow-up.

Influences of cardiovascular risk factors, baseline renal function, and biomarker/RIFLE group allocation were assessed in Cox proportional-hazard regression models. We found significant effect of all biomarker and RIFLE group allocation on long-term survival $(P<.001$ for all biomarkers). Exemplary, adjusted survival probability with regard to follow-up time is illustrated in Figure 4, $B$, for NGAL/RIFLE groups.

Additional data are presented in Table E1 and Figure 2, $A-D$.

\section{DISCUSSION \\ Key Findings}

We studied a cohort of 200 patients undergoing cardiac surgery to determine the short-term and long-term prognosis of AKI identified by standard criteria and/or urinary kidney biomarkers immediately after cardiac surgery. We found that urinary kidney biomarkers identified approximately $60 \%$ more cases with likely subclinical AKI than RIFLE status alone. The diagnosis of subclinical AKI was supported by evidence of increased risk of RRT initiation and in-hospital mortality. We found that novel urinary biomarker(+) patients, and less pronounced so, proteinuria $(+)$ patients, also carried higher risk of 
TABLE 4. Cox regression analysis for primary and secondary end points

\begin{tabular}{|c|c|c|c|c|c|c|c|c|}
\hline Variable & $\frac{\text { Hazard ratio }}{(95 \% \text { CI })}$ & $\begin{array}{c}P \\
\text { value }\end{array}$ & $\begin{array}{c}\text { Hazard ratio } \\
(95 \% \mathrm{CI})\end{array}$ & $\begin{array}{c}P \\
\text { value }\end{array}$ & $\begin{array}{c}\text { Hazard ratio } \\
(95 \% \mathrm{CI})\end{array}$ & $\begin{array}{c}P \\
\text { value }\end{array}$ & $\frac{\text { Hazard ratio }}{(95 \% \mathrm{CI})}$ & $\begin{array}{c}P \\
\text { value }\end{array}$ \\
\hline \multicolumn{9}{|c|}{ In-hospital mortality or renal replacement therapy as dependent end point } \\
\hline Age & $1.06(1.01-1.12)$ & .015 & $1.10(1.00-1.21)$ & .032 & $1.05(0.95-1.17)$ & .357 & $1.00(0.94-1.08)$ & .901 \\
\hline Previous cardiac surgery & $0.90(0.32-2.52)$ & .840 & $2.53(0.29-22.35)$ & .403 & $0.99(0.50-1.87)$ & .979 & $0.79(0.18-3.57)$ & .761 \\
\hline CABG vs concomitant surgery & $0.73(0.28-1.93)$ & .525 & $0.57(0.13-2.56)$ & .460 & $1.27(0.16-9.97)$ & .819 & $4.96(1.22-20.19)$ & .025 \\
\hline Perioperative fluid balance & $1.00(0.99-1.00)$ & .017 & $1.00(0.99-1.00)$ & .517 & $1.00(0.99-1.00)$ & .769 & $1.00(0.99-1.00)$ & .424 \\
\hline $\begin{array}{r}N G A L(+) / R I F L E(-) \text { vs } \\
\text { NGAL(-)/RIFLE(-) }\end{array}$ & & & $7.18(1.52-33.93)$ & .013 & & & & \\
\hline $\begin{array}{r}N G A L(-) / R I F L E(+) \text { vs } \\
\text { NGAL(-)/RIFLE(-) }\end{array}$ & & & & & $16.79(1.85-152.40)$ & .012 & & \\
\hline $\begin{array}{r}N G A L(+) / R I F L E(+) \text { vs } \\
\text { NGAL(-)/RIFLE(-) }\end{array}$ & & & & & & & $100.45(19.14-527.29)$ & $<.001$ \\
\hline \multicolumn{9}{|c|}{ In-hospital mortality as dependent end point } \\
\hline Age & $1.06(0.99-1.13)$ & .08 & $1.12(0.98-1.28)$ & .105 & $1.11(0.90-1.37)$ & .321 & $1.01(0.90-1.13)$ & .866 \\
\hline Previous cardiac surgery & $0.99(0.52-1.93)$ & .994 & $1.37(0.43-4.34)$ & .589 & $1.29(0.62-3.21)$ & .487 & $0.91(0.33-2.49)$ & .853 \\
\hline CABG vs concomitant surgery & $0.73(0.22-2.42)$ & 607 & $0.58(0.90-3.74)$ & .566 & $0.48(0.16-14.30)$ & 672 & $3.77(0.63-22.70)$ & .148 \\
\hline Perioperative fluid balance & $0.99(0.99-1.00)$ & .007 & $1.00(0.99-1.00)$ & .726 & $1.00(0.99-1.01)$ & 665 & $0.99(0.99-1.00)$ & .181 \\
\hline $\begin{array}{l}N G A L(+) / R I F L E(-) \text { vs } \\
\quad \operatorname{NGAL}(-) / \operatorname{RIFLE}(-)\end{array}$ & & & $13.02(1.34-126.96)$ & .027 & & & & \\
\hline $\begin{array}{r}N G A L(-) / R I F L E(+) \text { vs } \\
\text { NGAL(-)/RIFLE(-) }\end{array}$ & & & & & $21.51(1.62-285.31)$ & .020 & & \\
\hline $\begin{array}{l}N G A L(+) / R I F L E(+) \text { vs } \\
\quad \operatorname{NGAL}(-) / \operatorname{RIFLE}(-)\end{array}$ & & & & & & & $61.66(6.70-543.48)$ & $<.001$ \\
\hline \multicolumn{9}{|c|}{ Major adverse kidney events as dependent end point } \\
\hline Age & $1.07(1.02-1.13)$ & .004 & $1.10(1.01-1.21)$ & .032 & $1.047(0.95-1.20)$ & .350 & $1.00(0.94-1.08)$ & .881 \\
\hline Previous cardiac surgery & $0.65(0.26-1.60)$ & .345 & $2.53(0.29-22.35)$ & .403 & $2.90(0.22-37.86)$ & .416 & $0.77(0.17-3.46)$ & .737 \\
\hline CABG vs concomitant surgery & $0.81(0.32-2.01)$ & 642 & $0.57(0.13-2.56)$ & .460 & $1.62(0.23-11.47)$ & .631 & $5.01(1.23-20.40)$ & .024 \\
\hline Perioperative fluid balance & $1.00(0.99-1.00)$ & .032 & $1.00(0.99-1.00)$ & .517 & $1.00(0.99-1.00)$ & .745 & $1.00(0.99-1.00)$ & .445 \\
\hline $\begin{array}{r}N G A L(+) / R I F L E(-) \text { vs } \\
\text { NGAL(-)/RIFLE }(-)\end{array}$ & & & $7.18(1.52-33.93)$ & .013 & & & & \\
\hline $\begin{array}{r}N G A L(-) / R I F L E(+) \text { vs } \\
\text { NGAL }(-) / \operatorname{RIFLE}(-)\end{array}$ & & & & & $43.38(5.52-340.87)$ & $<.001$ & & \\
\hline $\begin{array}{c}N G A L(+) / R I F L E(+) \text { vs } \\
\text { NGAL(-)/RIFLE(-) }\end{array}$ & & & & & & & $108.99(20.96-566.74)$ & $<.001$ \\
\hline
\end{tabular}

Model adjusted for age, type of surgery (coronary artery bypass graft vs concomittant surgery), previous cardiac surgery, and perioperative fluid balance 0 to 6 h. Group comparisons performed versus $\operatorname{NGAL}(-) / \operatorname{RIFLE}(-)$ as a reference group. All group comparisons were inserted into the model one after another to exclude interaction. $95 \% C I, 95 \%$ Confidence interval; $C A B G$, coronary artery bypass grafting; $N G A L$, neutrophil gelatinase associated lipocalin; $R I F L E$, risk injury failure end-stage renal disease classification.

in-hospital adverse outcomes than biomarker(-) patients independent of RIFLE status. Three percent to $11 \%$ of patients after a cardiac surgery procedure appear to be influenced by single-biomarker( + ), subclinical AKI. Moreover, patients who were biomarker( -$)$ and $\operatorname{RIFLE}(+)$, implying potential loss of renal function without evidence of acute tubular injury, showed intermediate outcomes. As expected, biomarker(+) and RIFLE(+) patients had worst outcomes regarding need for RRT initiation, mortality, and combined end points. The outcome pattern observed in all urinary kidney biomarker-based patient subgroups was similar regardless which urinary biomarker was used with significant overlap among urinary biomarkers. In contrast, CRP did not separate patient outcomes beyond association with RIFLE status and urinary kidney biomarker grouping did not overlap with CRP-based patient groups. Finally, during long-term follow-up over 5 years, patient survival was associated with RIFLE status but also with early kidney biomarker status.

\section{Relationship With Previous Studies}

Pooled data from critically ill and cardiac surgery patients and data from 2 subsequent prospective studies enrolling patients treated in an emergency department assessed the short-term prognostic relevance of likely subclinical AKI (NGAL[+] and RIFLE[-] status). Such 

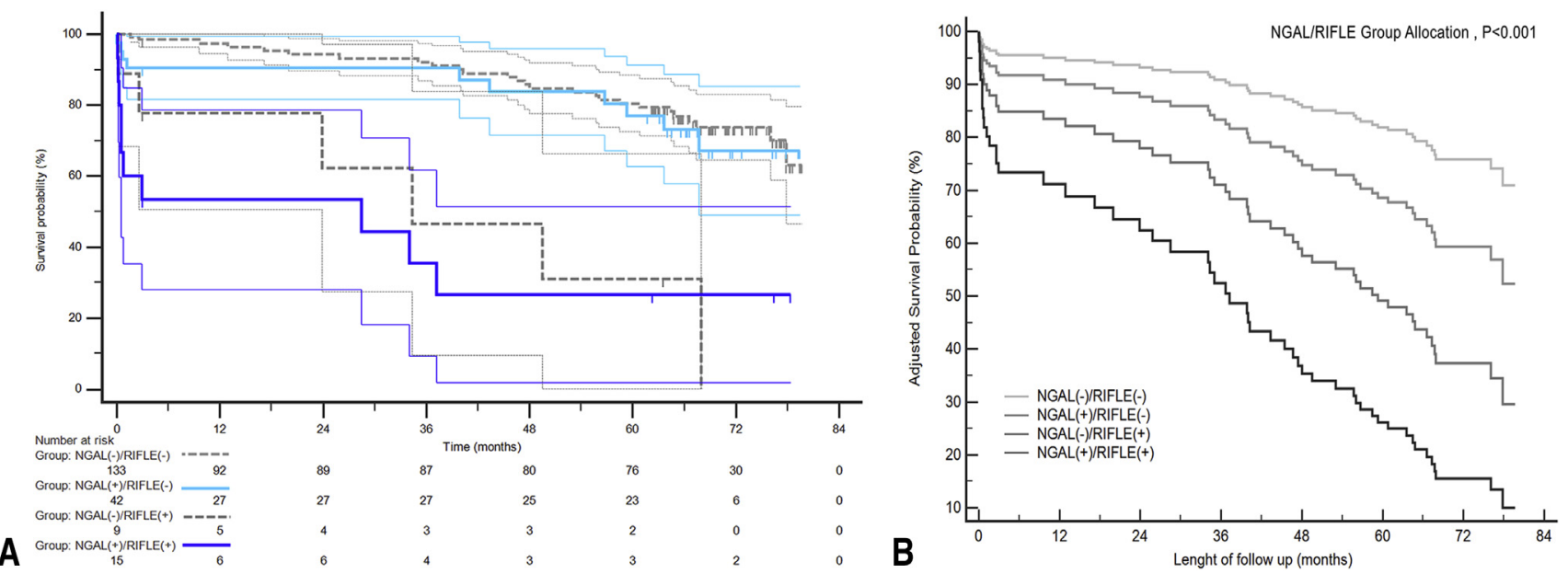

FIGURE 4. A, Kaplan-Meier survival curve showcasing neutrophil gelatinase-associated lipocalin/risk injury failure end-stage renal disease classification (NGAL/RIFLE) groups. Median follow up time was 5.6 years. Survival curves differ significantly early after cardiac surgery until the end of follow-up (All $P$ values $<.001$ for log-rank, Breslow test, and Tarone-Ware test). Graphs include the confidence limits as well as the tabled number of patients at risk periodically over time of follow-up. B, Multivariable-adjusted survival curves by NGAL/RIFLE group allocation. Survival curves are adjusted for preoperative creatinine value, peripheral vascular disease, arterial hypertension, and past history of myocardial infarction 6 months before surgery. NGAL/RIFLE group allocation $P<.001$.

patients were at increased risk of subsequent RRT and increased in-hospital mortality. ${ }^{4,6,7}$

The proportion of patients distributed among the biomarker/RIFLE subgroups were similar in our study cohort compared with these studies. ${ }^{4,6,7}$ The proportion of patients additionally identified as having subclinical AKI (biomarker[+]/RIFLE[-]) was higher in the present study (58\%-74\% depending on biomarker) compared with previous studies ( $40 \%-45 \%)$. Across studies, including the present study, in various clinical settings, prognostic pattern defined by urinary kidney biomarkers was similar-with best prognosis for kidney biomarker $(-) /$ RIFLE(-) patients and worst prognosis for kidney biomarker $(+) / \operatorname{RIFLE}(+)$ patients. More recently, Basu and colleagues ${ }^{27}$ demonstrated that a composite of a kidney injury biomarker and cystatin-C as glomerular filtration marker was superior in prediction of AKI than changes of creatinine in children undergoing cardiac surgery.

Only 1 study reported outcome pattern using more than one kidney biomarker ${ }^{6}$; however, overlap of patients between biomarker-defined subgroups was not assessed. None of the previous prospective studies reporting on urinary kidney biomarker/RIFLE status verified study results against nonkidney-related prognostic biomarkers such as C-reactive protein. Furthermore, no study has yet informed about RIFLE status and long-term outcomes. Our findings suggest partial translation of kidney biomarker-defined short-term outcomes to long-term outcomes. A causal relationship between early postoperative biomarker-detected tubular injury and long-term kidney prognosis may exist, as reported by Cooper and colleagues. ${ }^{28}$

\section{Implications of the Study Findings}

To the best of our knowledge, this is the first study to investigate on short- and long-term outcomes, including RRT, mortality, and composite end points according to urinary biomarker- and RIFLE-based kidney status in patients immediately after cardiac surgery. Our study implies that biomarkers of kidney injury identify a population at clearly increased risk of renal and clinical adverse events. Such renal impairment is not detected by routine clinical measurements (ie, creatinine and urine output) and should logically be considered to define a state of subclinical AKI. Patients with subclinical AKI subsequently developing positive RIFLE-criteria based AKI may be regarded as preclinical AKI diagnosed early by positive kidney biomarker status. Also, our study implies that such findings are minimally affected by the biomarker used and are likely conceptually robust. Moreover, the verification of data against the prognostic discriminative ability of a routine inflammatory marker (ie, CRP) implies a level of kidney specificity for our observations referring to the ability of NGAL and other biomarkers to detect decline in kidney function before changes in serum creatinine are seen. ${ }^{8}$

Subclinical AKI (without RIFLE positivity developing over the first postoperative week) and early biomarkeridentified AKI (preclinical AKI with subsequent postoperative RIFLE positivity) were detected within several hours after cardiac surgery, implying the ability to deliver early prognosis and, in future, early riskstratified intervention. ${ }^{29}$ Implementation of the KDIGO care bundles $^{30}$ (ie, avoidance of nephrotoxic agents, discontinuation of angiotensin-converting enzyme inhibitors and 


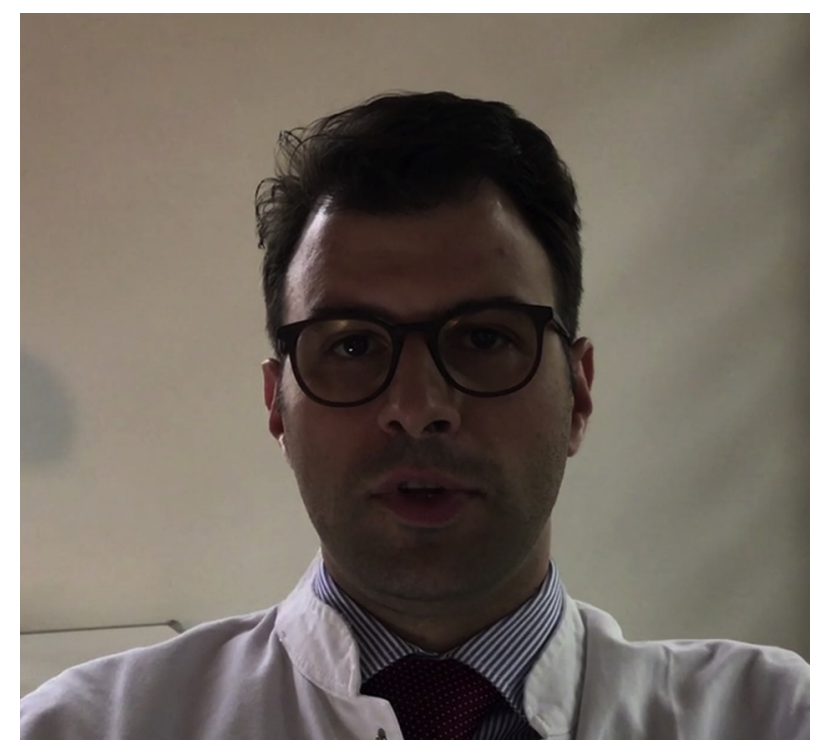

VIDEO 1. Christian Albert, MD, comments on the key study findings. Early postoperative measurement of urinary kidney biomarkers has important clinical implications for patient risk assessment. Positive urinary kidney-biomarker test results after cardiac surgery carries relevant prognostic information and identifies acute kidney injury subtypes with incrementally limited outcome. Video available at: http://www. jtcvsonline.org/article/S0022-5223(17)33020-9/fulltext.

angiotensin II receptor blockers for the first 48 hours after surgery, optimization of volume status, and hemodynamic parameters $^{31}$ ) and goal-directed patient individualized protocols $^{32}$ compared with standard care reduced the frequency and severity of AKI after cardiac surgery. In our cohort, the condition of clinical AKI without positive biomarker test results may cautiously be interpreted as a prerenal AKI subgroup, supported by more severely impaired cardiac dysfunction but not by signs of hypovolemia.

Finally, our study cautiously implies that there is a single biomarker-positive, subclinical AKI phenotype affecting up to $10 \%$ of cardiac surgical patients, a potentially novel category of AKI that may deserve further attention.

\section{Study Strengths and Limitations}

Our study reported on outcome pattern using more than 1 kidney biomarker ${ }^{6}$ and quantified overlap of patients between biomarker-defined subgroups. Also, we verified study results against nonkidney-related prognostic biomarkers such as CRP, assessed biomarkers against multiple relevant outcomes, and provided long-term follow-up.

The outcome of this analysis critically depends on the current creatinine- and urine output-based definition of AKI that is consensus-driven, established in clinical routine, and nonverifiable because there is no real-time glomerular filtration rate monitoring available. We cannot exclude type II error and potential study bias due to limited patient access of the initial multicenter study. However, nonparticipating study centers did not collect enough urine samples for measurement of additional biomarkers. Overall rate of patients developing RIFLE-AKI after cardiac surgical procedure in our cohort $12 \%(n=24)$ resembles a rather typical proportion with this postoperative complication $^{21}$; however, the study sample size and event rate limited our ability to perform combinatorial biomarker sensitivity analyses. The internal validity of our findings is strengthened by similar outcome patterns of 3 independent urine kidney biomarkers, exclusion of preoperative AKI, and choice of cardiac surgery patients at increased renal risk enabling concept evaluation in a relatively homogenous, well-defined patient cohort with assessable timing of AKI onset.

Finally, biomarker measurement at 6 hours after commencement of CPB may not reflect the point of best discriminatory power of these markers. ${ }^{13}$ Nonetheless, findings appeared to be robust and consistent.

The present study does not preclude the conclusion that no other urinary biomarkers may improve AKI risk prediction as previously shown. ${ }^{33}$ However, so far no combined assessment of glomerular (RIFLE) and tubular (dys)function on short- and long-term outcomes has been reported. Our study was initiated before studies reporting on the predictive ability of cell cycle arrest for adverse outcome in patients with AKI. ${ }^{34}$ Yet, such biomarkers will require assessment in terms of long-term prognosis. Unfortunately, short-term outcome for patients with AKI was influenced by high mortality rates and long-term data acquisition were compromised by 53 patients $(26.5 \%)$ being lost to follow-up. The number of surviving patients was too low and urine protein excretion was rarely recorded during ambulatory patients' care, limiting our long-term analysis by incomplete CKD detection and limited predictive value of urinary kidney biomarkers for the development of worsening CKD. These drawbacks could be improved in future studies by larger patient numbers and follow-up examination at the study center.

\section{CONCLUSIONS}

This hypothesis-generating substudy of an RCT found that early postoperative measurement of urinary kidney biomarkers has important clinical implications for patient risk assessment and long-term prognosis. In patients undergoing cardiac surgery, early postoperative urinary biomarker positivity added prognostic information regardless of RIFLE-AKI. Thus, our study provides new evidence that urinary kidney biomarkers identify AKI subtypes with incrementally worsening prognosis and may therefore define a state of clinically relevant subclinical AKI (Video 1). 


\section{Conflict of Interest Statement}

R.B. has acted as paid consultant to Abbott Diagnostics and Biosite Inc. A.H.-F. has received honoraria for speaking for Abbott Diagnostics, Alere, and Biosite Inc. C.A. has received honoraria for speaking for Siemens Healthcare Diagnostics and several companies are involved in the development of the neutrophil gelatinase-associated lipocalin assays for application in clinical practice. All other authors have nothing to disclose with regard to commercial support.

The authors thank Professor Siegfried Kropf, Institute for Biometrics and Medical Informatics, Otto-von-Guericke University, Magdeburg, Germany, for his advice and substantive comments on an earlier draft of the manuscript.

\section{References}

1. Haase M, Bellomo R, Matalanis G, Calzavacca P, Dragun D, Haase-Fielitz A. A comparison of the RIFLE and Acute Kidney Injury Network classifications for cardiac surgery-associated acute kidney injury: a prospective cohort study. J Thorac Cardiovasc Surg. 2009;138:1370-6.

2. Kim K, Joung K-W, Ji S-M, Kim JY, Lee EH, Chung CH, et al. The effect of coronary angiography timing and use of cardiopulmonary bypass on acute kidney injury after coronary artery bypass graft surgery. J Thorac Cardiovasc Surg. 2016;152:254-61.e3.

3. Chen SW, Tsai FC, Lin YS, Chang CH, Chen DY, Chou AH, et al. Long-term outcomes of extracorporeal membrane oxygenation support for postcardiotomy shock. J Thorac Cardiovasc Surg. 2017;154:1-12.

4. Haase M, Devarajan P, Haase-Fielitz A, Bellomo R, Cruz DN, Wagener G, et al The outcome of neutrophil gelatinase-associated lipocalin-positive subclinical acute kidney injury. J Am Coll Cardiol. 2011;57:1752-61.

5. Haase M, Kellum JA, Ronco C. Subclinical AKI-an emerging syndrome with important consequences. Nat Rev Nephrol. 2012;8:735-9.

6. Nickolas TL, Schmidt-Ott KM, Canetta P, Forster C, Singer E, Sise M, et al. Diagnostic and prognostic stratification in the emergency department using urinary biomarkers of nephron damage. J Am Coll Cardiol. 2012;59:246-55.

7. Di Somma S, Magrini L, De Berardinis B, Marino R, Ferri E, Moscatelli P, et al. Additive value of blood neutrophil gelatinase- associated lipocalin to clinical judgment in acute kidney injury diagnosis and mortality prediction in patients hospitalized from the emergency department. Crit Care. 2013;17:R29.

8. McCullough PA, Shaw AD, Haase M, Bouchard J, Waikar SS, Siew ED, et al. Diagnosis of acute kidney injury using functional and injury biomarkers: workgroup statements from the tenth Acute Dialysis Quality Initiative Consensus Conference. Contrib Nephrol. 2013;182:13-29.

9. Huen SC, Parikh CR. Molecular phenotyping of clinical AKI with novel urinary biomarkers. Am J Physiol Renal Physiol. 2015;309:F406-13.

10. Dennen P, Altmann C, Kaufman J, Klein CL, Andres-Hernando A, Ahuja NH, et al. Urine interleukin-6 is an early biomarker of acute kidney injury in children undergoing cardiac surgery. Crit Care. 2010;14:R181.

11. Renhua L, Miaolin C, Junlin W, Qingwei W, Xiaoping X, Huili D, et al. The level of the biomarkers at the time of nephrology consultation might predict the prognosis of acute kidney injury in hospitalized patients. Blood Purif. 2014;38:89-95.

12. Sato W, Sato Y. Midkine in nephrogenesis, hypertension and kidney diseases. Br J Pharmacol. 2014;171:879-87.

13. Hayashi H, Sato W, Kosugi T, Nishimura K, Sugiyama D, Asano N, et al. Efficacy of urinary midkine as a biomarker in patients with acute kidney injury. Clin Exp Nephrol. 2016;21:1-11.

14. Haase M, Bellomo R, Devarajan P, Schlattmann P, Haase-Fielitz A, NGAL Meta-analysis Investigator Group. Accuracy of neutrophil gelatinaseassociated lipocalin (NGAL) in diagnosis and prognosis in acute kidney injury: a systemic review and meta-analysis. AJKD. 2009;54:1012-24.

15. Zappitelli M, Coca SG, Garg AX, Krawczeski CD, Thiessen Heather P, Sint K, et al. The association of albumin/creatinine ratio with postoperative AKI in children undergoing cardiac surgery. Clin J Am Soc Nephrol. 2012;7:1761-9.
16. Li S-Y, Chuang C-L, Yang W-C, Lin S-J. Proteinuria predicts postcardiotomy acute kidney injury in patients with preserved glomerular filtration rate. J Thorac Cardiovasc Surg. 2015;149:894-9.

17. Kangasniemi OP, Biancari F, Luukkonen J, Vuorisalo S, Satta J, Pokela R, et al. Preoperative C-reactive protein is predictive of long-term outcome after coronary artery bypass surgery. Eur J Cardiothorac Surg. 2006;29:983-5.

18. van Straten AHM, Soliman Hamad MA, van Zundert AJ, Martens EJ, Schönberger JPAM, de Wolf AM. Preoperative C-reactive protein levels to predict early and late mortalities after coronary artery bypass surgery: eight years of follow-up. J Thorac Cardiovasc Surg. 2009;138:954-8.

19. Haase M, Haase-Fielitz A, Plass M, Kuppe H, Hetzer R, Hannon C, et al. Prophylactic perioperative sodium bicarbonate to prevent acute kidney injury following open heart surgery: a multicenter double-blinded randomized controlled trial. PLoS Med. 2013;10:e1001426.

20. Bellomo R, Ronco C, Kellum JA, Mehta RL, Palevsky P. Acute renal failure definition, outcome measures, animal models, fluid therapy and information technology needs: the Second International Consensus Conference of the Acute Dialysis Quality Initiative (ADQI) Group. Crit Care. 2004;8: R204-12.

21. Englberger L, Suri RM, Li Z, Casey ET, Daly RC, Dearani JA, et al. Clinical accuracy of RIFLE and Acute Kidney InjuryNetwork (AKIN) criteria for acute kidney injury in patients undergoing cardiac surgery. Crit Care. 2011;15:R16.

22. Xiong J, Tang X, Hu Z, Nie L, Wang Y, Zhao J. The RIFLE versus AKIN classification for incidence and mortality of acute kidney injury in critical ill patients: A meta-analysis. Sci Rep. 2015;5:1-9.

23. KDIGO 2012 clinical practice guideline for the evaluation and management of chronic kidney disease. Kidney Int Suppl. 2013;3:5-14.

24. Youden WJ. Index for rating diagnostic tests. Cancer. 1950;3:32-5.

25. Landis JR, Koch GG. The measurement of observer agreement for categorical data. Biometrics. 1977;33:159-74.

26. Thakar CV. A clinical score to predict acute renal failure after cardiac surgery. $J$ Am Soc Nephrol. 2004;16:162-8.

27. Basu RK, Wong HR, Krawczeski CD, Wheeler DS, Manning PB, Chawla LS, et al. Combining functional and tubular damage biomarkers improves diagnostic precision for acute kidney injury after cardiac surgery. J Am Coll Cardiol. 2014; 64:2753-62.

28. Cooper DS, Claes D, Goldstein SL, Bennett MR, Ma Q, Devarajan P, et al. Follow-Up Renal Assessment of Injury Long-Term After Acute Kidney Injury (FRAIL-AKI). Clin J Am Soc Nephrol. 2016;11:21-9.

29. Yi Ng S, Sanagou M, Wolfe R, Cochrane A, Smith JA, Reid CM. Prediction of acute kidney injury within 30 days of cardiac surgery. J Thorac Cardiovasc Surg. 2014;147:1875-83.e1.

30. Meersch M, Schmidt C, Hoffmeier A, Van Aken H, Wempe C, Gerss J, et al. Pre vention of cardiac surgery-associated AKI by implementing the KDIGO guidelines in high risk patients identified by biomarkers: the PrevAKI randomized controlled trial. Intensive Care Med. 2017;43:1-11.

31. Haase-Fielitz A, Haase M, Bellomo R, Calzavacca P, Spura A, Baraki H, et al. Perioperative hemodynamic instability and fluid overload are associated with increasing acute kidney injury severity and worse outcome after cardiac surgery. Blood Purif. 2017;43:298-308.

32. Magruder JT, Crawford TC, Harness HL, Grimm JC, Suarez-Pierre A, Wierschke C, et al. A pilot goal-directed perfusion initiative is associated with less acute kidney injury after cardiac surgery. J Thorac Cardiovasc Surg. 2017; 153:118-25.e1.

33. de Melo Bezerra Cavalcante CT, Magalhães Castelo Branco K, Cavalcante Pinto Júnior V, et al. Syndecan-1 improves severe acute kidney injury prediction after pediatric cardiac surgery. J Thorac Cardiovasc Surg. 2016;152: 178-86.e2.

34. Koyner JL, Shaw AD, Chawla LS, Hoste EA, Bihorac A, Kashani K, et al. Tissue inhibitor metalloproteinase-2 (TIMP-2) IGF-binding protein-7 (IGFBP7) Levels are associated with adverse long-term outcomes in patients with AKI. J Am Soc Nephrol. 2015;26:1747-54.

Key Words: acute kidney injury, cardiac surgery, neutrophil gelatinase-associated lipocalin, midkine, interleukin-6, subclinical AKI 


\section{RESULTS}

CRP and controlling outcome pattern for systemic inflammation, baseline and perioperative characteristics, biomarker courses, and group status for CRP and consecutive patient adverse outcomes (Tables E6 and E7); and using CRP levels at 6 hours after commencement of cardiopulmonary bypass, no additive prognostic information for the primary end point, in-hospital mortality, renal replacement therapy (RRT) or major adverse kidney events (MAKEs) was found beyond RIFLE status in adjusted and unadjusted models (Figure 3, A, and Table E16). Prognostic patterns and biomarker agreement over acute kidney injury (AKI) subtypes using CRP-concentrations measured at 24 hours remained essentially unchanged (Figure 3, B).
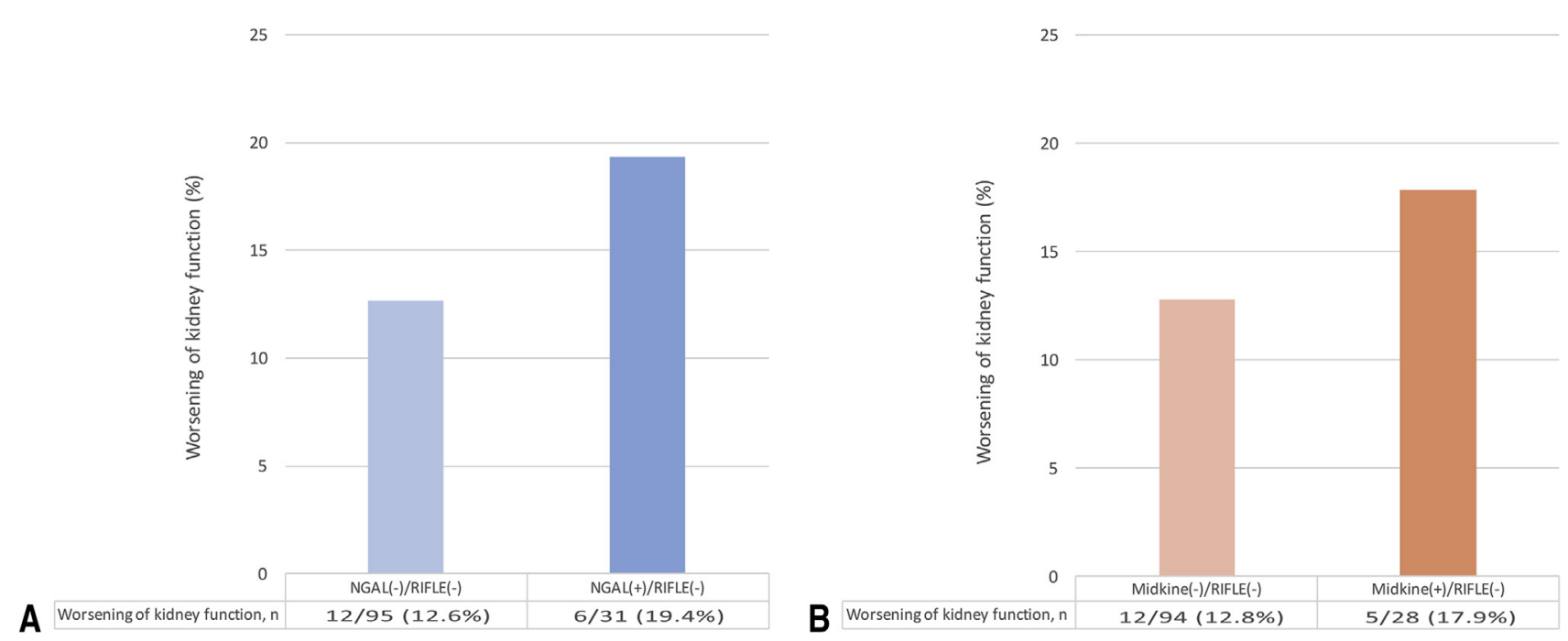

FIGURE E1. Overall, 42 of 105 patients (40.0\%) developed worsening of chronic kidney disease (CKD), but no patient developed dependence on chronic hemodialysis. Patients with neutrophil gelatinase-associated lipocalin (NGAL)-positive or midkine-positive subclinical acute kidney injury showed a nonsignificant increase in the chance of developing worsening CKD compared with NGAL-negative/renal risk, injury, failure, loss of renal function, end-stage renal disease classification (RIFLE)-negative patients (odds ratio, 1.66; 95\% confidence interval, 0.57-4.87; $P=.381$ ) $($ Figure $1, A)$ or midkine-negative/RIFLE-negative patients (odds ratio, 1.49; 95\% confidence interval, 0.47-4.65; $P=.537$ ) (Figure E1, $B$ ), respectively. Long-term worsening CKD according to biomarker status. A, Proportion of NGAL-positive/RIFLE-negative patients developing worsening CKD during follow-up period of 5.6 years compared with NGAL-negative/RIFLE-negative patients. B, Proportion of midkine-positive/RIFLE-negative patients developing worsening CKD during follow-up period of 5.6 years compared with midkine-negative/RIFLE-negative patients. ${ }^{16}$ 

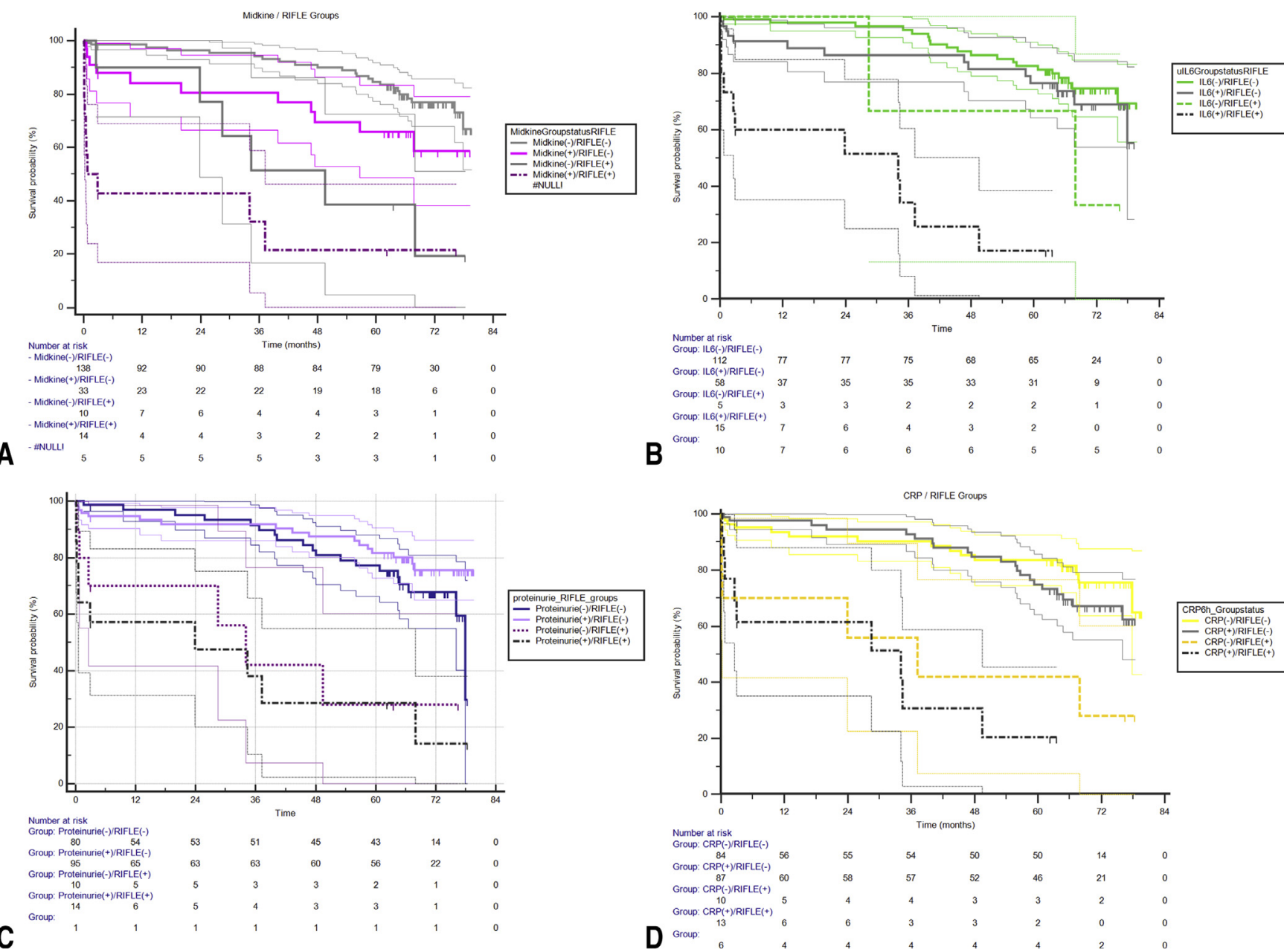

FIGURE E2. A-D, Kaplan-Meier survival curves for midkine, interleukin-6 (IL-6), proteinuria, and C-reactive protein $(C R P)$ groups. Kaplan-Meier survival curves illustrate significant differences for all assessed biomarker/renal risk, injury, failure, loss of renal function, end-stage renal disease classification (RIFLE) groups. For all biomarkers assessed, survival distribution between the groups differed significantly early after cardiac surgery as well as for short-term and longterm follow-up as indicated by log-rank test, Breslow test, and Tarone-Ware test considering (weighted) left, middle, and right parts of the Kaplan-Meier curve (all $P$ values $<.001$ ). Graphs include the confidence limits as well as the number of patients at risk periodically over time of follow-up. 
TABLE E1. Inclusion and exclusion criteria of the original randomized controlled trial (ClinicalTrials.gov No. NCT00672334) ${ }^{19}$

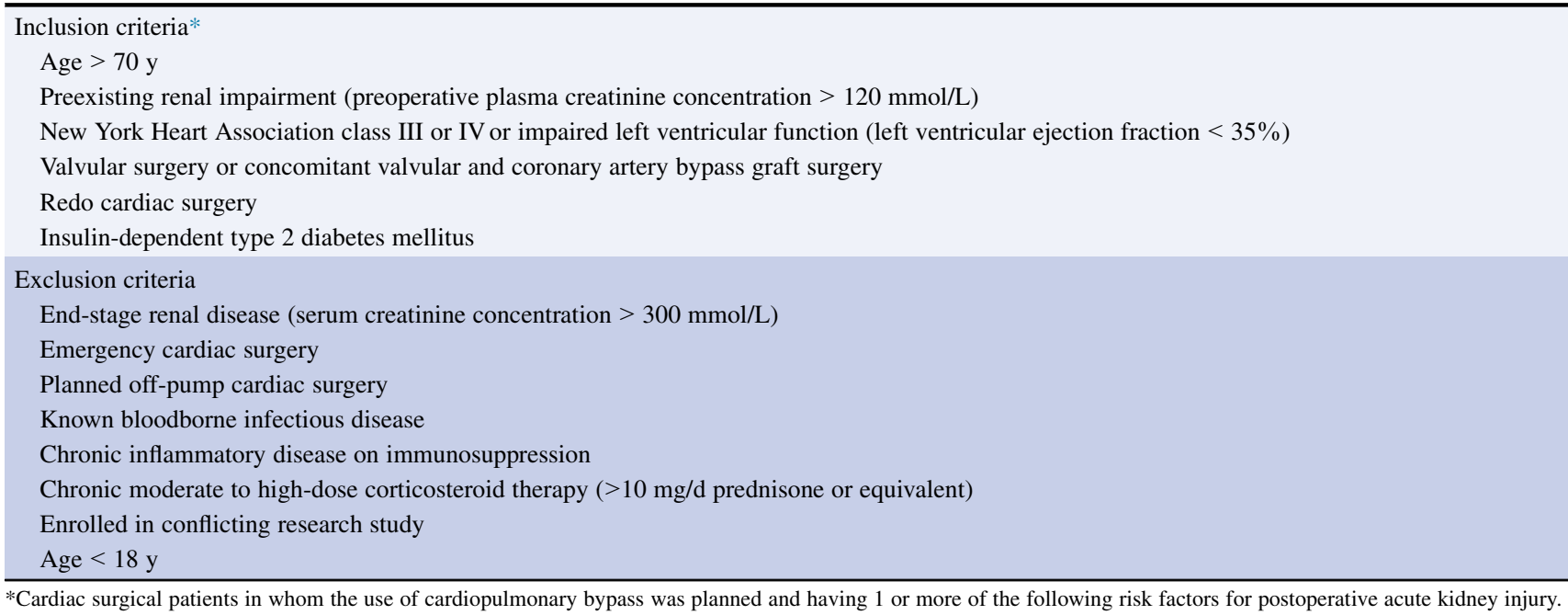

TABLE E2. Preoperative patient characteristics according to midkine/renal risk, injury, failure, loss of renal function, end stage renal disease classification (RIFLE) subgroup $^{20}$

\begin{tabular}{|c|c|c|c|c|c|}
\hline Variable & $\frac{\text { Midkine }(-) / \operatorname{RIFLE}(-)}{(\mathrm{n}=138 ; 70.8 \%)}$ & $\frac{\text { Midkine }(+) / \text { RIFLE }(-)}{(\mathbf{n}=33 ; 16.9 \%)}$ & $\frac{\text { Midkine }(-) / \operatorname{RIFLE}(+)}{(\mathbf{n}=10 ; 5.1 \%)}$ & $\frac{\text { Midkine }(+) / \text { RIFLE }(+)}{(n=14 ; 7.2 \%)}$ & $\begin{array}{c}P \\
\text { value }\end{array}$ \\
\hline Age $(y)$ & $67(58-72)$ & $73(64-78)$ & $73.5(69-77.5)$ & $71.5(66.5-77)$ & .003 \\
\hline Female sex & $40(29)$ & $10(30.3)$ & $2(20)$ & $6(42.9)$ & .669 \\
\hline Body mass index & $26.2(23.4-29.3)$ & $26.1(24.6-28.1)$ & $27.9(26-32.5)$ & $26(24.5-31.4)$ & .051 \\
\hline Insulin-dependent diabetes mellitus & $8(5.8)$ & $1(3)$ & $0(0)$ & $0(0)$ & 1.000 \\
\hline Noninsulin-dependent diabetes mellitus & $28(20.3)$ & $5(15.2)$ & $1(10)$ & 4 (28.6) & .667 \\
\hline Arterial hypertension & $102(73.9)$ & $25(75.8)$ & $10(100)$ & $13(92.9)$ & .138 \\
\hline Hypercholesterolemia & $89(64.5)$ & $20(60.6)$ & $8(80)$ & $9(64.3)$ & .792 \\
\hline Chronic obstructive pulmonary disease & $19(13.8)$ & $6(18.2)$ & $2(20)$ & $3(21.4)$ & .672 \\
\hline Current smoking habit & $21(15.2)$ & $4(12.1)$ & $3(30)$ & $1(7.1)$ & .471 \\
\hline Preoperative serum creatinine $(\mu \mathrm{mol} / \mathrm{L})$ & $88.4(76-106.1)$ & $88.4(79.6-106.1)$ & $114.5(88.2-123.8)$ & $88.4(73-118.7)$ & .114 \\
\hline Peripheral vascular disease & $31(22.5)$ & $10(30.3)$ & $4(40)$ & $4(28.6)$ & .455 \\
\hline Left ventricular dysfunction* & 27 (19.6) & $6(18.2)$ & $3(30)$ & $4(28.6)$ & .635 \\
\hline Left ventricular ejection fraction $(\%)$ & $55(40-60)$ & $50(40-60)$ & $47(30-56)$ & $57.5(45-60)$ & .519 \\
\hline \multicolumn{6}{|l|}{ Preoperative medication } \\
\hline ACE inhibitor/AT-II antagonist & $75(54.3)$ & $19(57.6)$ & $6(60)$ & $12(85.7)$ & .161 \\
\hline Beta-blocker & 99 (71.7) & $25(75.8)$ & $6(60)$ & $12(85.7)$ & .523 \\
\hline Calcium channel blocker & $39(28.3)$ & $10(30.3)$ & $5(50)$ & $5(35.7)$ & .468 \\
\hline HMG-CoA reductase inhibitor & $81(58.7)$ & $16(48.5)$ & $8(80)$ & $7(50)$ & .312 \\
\hline Diuretics & $87(63.0)$ & $21(63.6)$ & $8(80)$ & $12(85.7)$ & .301 \\
\hline
\end{tabular}

Values are presented as median (25th-75th percentile) or frequency (\%). RIFLE, Risk injury failure end-stage renal disease classification; $A C E$, angiotensin-converting enzyme; AT-II, angiotensin-II; HMG-CoA, 3-hydroxy-3-methyl-glutaryl-coenzyme A. *Left ventricular ejection fraction $<35 \%$. 
TABLE E3. Perioperative patient characteristics according to midkine/renal risk, injury, failure, loss of renal function, end stage renal disease classification (RIFLE) subgroup $^{20}$

\begin{tabular}{|c|c|c|c|c|c|}
\hline Variable & $\frac{\text { Midkine }(-) / \operatorname{RIFLE}(-)}{(\mathrm{n}=138 ; 70.8 \%)}$ & $\frac{\text { Midkine(+)/RIFLE }(--)}{(n=33 ; 16.9 \%)}$ & $\frac{\text { Midkine }(-) / \text { RIFLE }(+)}{(n=10 ; 5.1 \%)}$ & $\frac{\text { Midkine }(+) / \operatorname{RIFLE}(+)}{(\mathrm{n}=14 ; 7.2 \%)}$ & $\begin{array}{c}P \\
\text { value }\end{array}$ \\
\hline \multicolumn{6}{|l|}{ Procedures } \\
\hline CABG surgery & $29(21)$ & $1(3)$ & $3(30)$ & $1(7.1)$ & .023 \\
\hline Valvular surgery & $64(46.4)$ & $12(36.4)$ & $3(30)$ & $9(64.3)$ & .260 \\
\hline $\mathrm{CABG}$ and valvular surgery & $35(25.4)$ & $12(36.4)$ & $3(30)$ & $3(21.4)$ & .591 \\
\hline Thoracic aortic surgery & $9(6.5)$ & $8(24.2)$ & $0(0)$ & $1(7.1)$ & .024 \\
\hline Ventricular assist device & $1(0.7)$ & $0(0)$ & $1(10)$ & $0(0)$ & .157 \\
\hline Redo cardiac surgery & $31(22.5)$ & $10(30.3)$ & $5(50)$ & $5(35.7)$ & .229 \\
\hline $\begin{array}{l}\text { Duration of cardiopulmonary } \\
\text { bypass (min) }\end{array}$ & $118(91-140)$ & $146(113-222)$ & $149(91-173)$ & $227(142-370)$ & $<.001$ \\
\hline $\begin{array}{l}\text { Lowest intraoperative } \\
\text { MAP (mm Hg) }\end{array}$ & $34.5(31-40)$ & $32(27-40)$ & $31.5(24-35.5)$ & $31(23-41.2)$ & .452 \\
\hline \multicolumn{6}{|l|}{$\begin{array}{l}\text { Perioperative fluid balance and } \\
\text { medication }(0-24 \mathrm{~h})\end{array}$} \\
\hline Fluid intake (mL) & 8227 (6800-9709) & 8456 (6493-9734) & $6481(5677-8191)$ & $5731(3496-7449)$ & .001 \\
\hline Urine output (mL) & $5470(4586-6455)$ & $4890(4317-6782)$ & $4040(2678-4246)$ & $3836(3064-4620)$ & $<.001$ \\
\hline Packed red blood cells (mL) & $250(0-500)$ & $500(0-1000)$ & $525(0-1437)$ & $1125(438-2688)$ & .005 \\
\hline Drain output $(\mathrm{mL})$ & $459(350-675)$ & $650(472-962)$ & $672(214-1525)$ & $1010(688-2243)$ & $<.001$ \\
\hline Fluid balance (mL) & $2766(1727-3851)$ & 2902 (1000-5147) & $3592(2496-5024)$ & $1450(-119$ to 2843$)$ & .131 \\
\hline Furosemide (mg) & $20(8.75-40)$ & $20(5-40)$ & $65(20-242)$ & $60(20-113)$ & $<.001$ \\
\hline \multicolumn{6}{|l|}{ Urine midkine (pg/mL) } \\
\hline $0 \mathrm{~h}$ & $94.4(48.9-147.5)$ & $88(51.8-195.3)$ & $58.6(20.9-109.8)$ & $97.7(43.4-217.3)$ & .361 \\
\hline $6 \mathrm{~h}$ & $101.3(73.9-161)$ & 474 (313-1800) & $114.5(63.5-151.2)$ & $1127.5(431.3-3502.4)$ & $<.001$ \\
\hline $24 \mathrm{~h}$ & $77.3(49.1-102)$ & $104.5(55.3-144)$ & $43.1(28.8-278.7)$ & $98.9(63.9-270.9)$ & .147 \\
\hline RIFLE stage & & & & & .862 \\
\hline Risk & & & $6(60)$ & $7(50)$ & \\
\hline Injury & & & $3(30)$ & $4(28.6)$ & \\
\hline Failure & & & $1(10)$ & $3(21.4)$ & \\
\hline
\end{tabular}

Values are presented as median (25th-75th percentile) or frequency (\%). RIFLE, Risk injury failure end-stage renal disease classification; CABG, coronary artery bypass graft; $M A P$, mean arterial pressure. 
TABLE E4. Preoperative patient characteristics according to interlukin-6/renal risk, injury, failure, loss of renal function, end stage renal disease classification (IL-6/RIFLE) subgroup ${ }^{20}$

\begin{tabular}{|c|c|c|c|c|c|}
\hline Variable & $\frac{\text { IL-6 }(-) / \text { RIFLE }(-)}{(n=112 ; 58.9 \%)}$ & $\frac{\operatorname{IL6}(+) / \operatorname{RIFLE}(-)}{(\mathrm{n}=58 ; 30.5 \%)}$ & $\frac{\operatorname{IL-6}(-) / \operatorname{RIFLE}(+)}{(\mathrm{n}=5 ; 2.6 \%)}$ & $\frac{\text { IL-6 }(+) / \text { RIFLE }(+)}{(n=15 ; 7.9 \%)}$ & $\begin{array}{c}P \\
\text { value }\end{array}$ \\
\hline Age (y) & $68(56-72)$ & $70(62.7-76.3)$ & $76(69-77.5)$ & $72(69-75)$ & .016 \\
\hline Female sex & $38(33.9)$ & $13(22.4)$ & $1(20)$ & $4(26.7)$ & .457 \\
\hline Body mass index & $26(23.4-29.2)$ & $26.8(24.6-29.3)$ & $27(24.8-38.7)$ & $28(24.6-31.3)$ & .141 \\
\hline Insulin-dependent diabetes mellitus & $6(5.4)$ & $3(5.2)$ & $0(0)$ & $0(0)$ & .738 \\
\hline Noninsulin-dependent diabetes mellitus & $22(19.6)$ & $12(20.7)$ & $1(20)$ & $3(20)$ & 1.000 \\
\hline Arterial hypertension & $85(75.9)$ & $40(69)$ & $5(100)$ & $14(93.3)$ & .155 \\
\hline Hypercholesterolemia & $75(67)$ & $35(60.3)$ & $4(80)$ & $10(66.7)$ & .781 \\
\hline Chronic obstructive pulmonary disease & $15(13.4)$ & $11(19)$ & $1(20)$ & $4(26.7)$ & .388 \\
\hline Current smoking habit & $14(12.5)$ & $12(20.7)$ & $1(20)$ & $2(13.3)$ & .449 \\
\hline Preoperative serum creatinine $(\mu \mathrm{mol} / \mathrm{L})$ & $88.4(75.3-106.1)$ & $87(77.8-106.0)$ & $123.8(90.6-128.2)$ & $88.4(85.8-123.8)$ & .139 \\
\hline Peripheral vascular disease & $31(27.7)$ & $12(20.7)$ & $2(40)$ & $6(40)$ & .345 \\
\hline Left ventricular dysfunction* & $20(17.9)$ & $13(22.4)$ & $2(40)$ & $5(33.3)$ & .273 \\
\hline Left ventricular ejection fraction (\%) & $55(40-60)$ & $50(40-60)$ & $50(30-55)$ & $50(30-60)$ & .346 \\
\hline \multicolumn{6}{|l|}{ Preoperative medication } \\
\hline ACE inhibitor/AT-II antagonist & $57(50.9)$ & $36(62.1)$ & $3(60)$ & $13(86.7)$ & .039 \\
\hline Beta-blocker & $80(71.4)$ & $43(74.1)$ & $2(40)$ & $14(93.3)$ & .102 \\
\hline Calcium channel blocker & $32(28.6)$ & $17(29.3)$ & $3(60)$ & $5(33.3)$ & .517 \\
\hline HMG-CoA reductase inhibitor & $67(59.8)$ & $31(53.4)$ & $4(80)$ & $8(53.3)$ & .657 \\
\hline Diuretics & $64(57.1)$ & $40(69)$ & $3(60)$ & $13(86.7)$ & .087 \\
\hline
\end{tabular}

Values are presented as median (25th-75th percentile) or frequency (\%). IL-6, Interleukin 6; RIFLE, risk injury failure end-stage renal disease classification; $A C E$, angiotensin-converting enzyme; AT-II, angiotensin-II; HMG-CoA, 3-hydroxy-3-methyl-glutaryl-coenzyme A. *Left ventricular ejection fraction $<35 \%$. 
TABLE E5. Perioperative patient characteristics according to interlukin-6/renal risk, injury, failure, loss of renal function, end-stage renal disease classification (IL-6/RIFLE) subgroup ${ }^{20}$

\begin{tabular}{|c|c|c|c|c|c|}
\hline Variable & $\frac{\text { IL-6(-)/RIFLE }(-)}{(n=112 ; 58.9 \%)}$ & $\frac{\operatorname{IL6}(+) / \text { RIFLE }(-)}{(\mathbf{n}=58 ; 30.5 \%)}$ & $\frac{\text { IL-6(-)/RIFLE }(+)}{(\mathbf{n}=5 ; 2.6 \%)}$ & $\frac{\text { IL-6(+)/RIFLE }(+)}{(n=15 ; 7.9 \%)}$ & $\begin{array}{c}P \\
\text { value }\end{array}$ \\
\hline \multicolumn{6}{|l|}{ Procedure } \\
\hline CABG surgery & $23(20.5)$ & $9(15.5)$ & $2(40)$ & $2(13.3)$ & .466 \\
\hline Valvular surgery & $48(42.9)$ & $25(43.1)$ & $3(60)$ & $6(40)$ & .917 \\
\hline CABG and valvular surgery & $29(25.9)$ & $18(31)$ & $0(0)$ & $5(33.3)$ & .488 \\
\hline Thoracic aortic surgery & $11(9.8)$ & $6(10.3)$ & $0(0)$ & $1(6.7)$ & 1.000 \\
\hline Ventricular assist device & $1(0.9)$ & $0(0)$ & $0(0)$ & $1(6.7)$ & .292 \\
\hline Redo cardiac surgery & $23(20.5)$ & $17(29.3)$ & $3(60)$ & $4(26.7)$ & .144 \\
\hline Duration of cardiopulmonary bypass (min) & $115(88-138)$ & $137(105-180)$ & $117(81-267)$ & $168(129-246)$ & $<.001$ \\
\hline Lowest intraoperative MAP (mm Hg) & $36(31-41)$ & $33(28.8-38.2)$ & $34(32.5-40.5)$ & $31(24-39)$ & .454 \\
\hline \multicolumn{6}{|c|}{ Perioperative fluid balance and medication $(0-24 \mathrm{~h})$} \\
\hline Fluid intake (mL) & $8263(6819-9715)$ & $8109(6198-9671)$ & $6112(4215-8922)$ & $5856(4912-7362)$ & .002 \\
\hline Urine output $(\mathrm{mL})$ & $5468(4496-6485)$ & $5110(4213-6463)$ & $4020(1678-4188)$ & $3575(2880-4340)$ & $<.001$ \\
\hline Packed red blood cells (mL) & $250(0-500)$ & $500(0-750)$ & $500(0-1000)$ & $1000(250-2500)$ & .025 \\
\hline Drain output $(\mathrm{mL})$ & $460(331-675)$ & $562(400-806)$ & $219(200-515)$ & $1330(700-2125)$ & $<.001$ \\
\hline Fluid balance (mL) & $2727(1597-4106)$ & $2759(1564-4069)$ & $4021(200-6108)$ & $2696(384-3426)$ & .673 \\
\hline Furosemide (mg) & $20(10-40)$ & $20(0-43)$ & $70(15-240)$ & $60(20-150)$ & .002 \\
\hline \multicolumn{6}{|l|}{ Urine IL-6 (pg/mL) } \\
\hline $0 \mathrm{~h}$ & $3.36(2.42-5.38)$ & $4.54(2.99-8.72)$ & $3.07(2.18-4.66)$ & $5.22(2.96-10.45)$ & .047 \\
\hline $6 \mathrm{~h}$ & $4.65(3.48-7.01)$ & $26.27(14.83-68.52)$ & $4.63(4.05-8.70)$ & $25.04(19.02-77.41)$ & $<.001$ \\
\hline $24 \mathrm{~h}$ & $6.58(3.66-12.12)$ & $12.10(5.65-23.74)$ & $8.89(3.99-11.31)$ & $13.29(5.18-23.23)$ & .007 \\
\hline RIFLE stage & & & & & 1.000 \\
\hline Risk & & & $3(60)$ & $10(66.7)$ & \\
\hline Injury & & & $1(20)$ & $3(20)$ & \\
\hline Failure & & & $1(20)$ & $2(13.3)$ & \\
\hline
\end{tabular}

Numbers denote median (25th-75th percentile) or frequency (\%) where appropriate. IL-6, Interleukin 6; RIFLE, risk injury failure end-stage renal disease classification; $C A B G$, coronary artery bypass graft; $M A P$, mean arterial pressure. 
TABLE E6. Preoperative patient characteristics according to C-reactive protein/renal risk, injury, failure, loss of renal function, end-stage renal disease classification (CRP/RIFLE) subgroup ${ }^{20}$

\begin{tabular}{|c|c|c|c|c|c|}
\hline Variable & $\frac{\operatorname{CRP}(-) / \operatorname{RIFLE}(-)}{(\mathrm{n}=84 ; 43.3 \%)}$ & $\frac{\operatorname{CRP}(+) / \operatorname{RIFLE}(-)}{(\mathrm{n}=87 ; 44.8 \%)}$ & $\frac{\operatorname{CRP}(-) / \text { RIFLE }(+)}{(\mathrm{n}=10 ; 5.2 \%)}$ & $\frac{\text { CRP }(+) / \text { RIFLE }(+)}{(n=13 ; 6.7 \%)}$ & $\begin{array}{c}P \\
\text { value }\end{array}$ \\
\hline Age (y) & $67(59.3-72.8)$ & $70(58-75)$ & $75.5(68.8-77.3)$ & $72(68-75.5)$ & .022 \\
\hline Female sex & $26(31)$ & $25(28.7)$ & $3(30)$ & $4(30.8)$ & .991 \\
\hline Body mass index & $26.3(23.6-28.7)$ & $26.2(23.4-30.3)$ & $25.5(24.5-30.4)$ & $29.2(26.1-35.1)$ & .017 \\
\hline Insulin-dependent diabetes mellitus & $4(4.8)$ & $5(5.7)$ & $0(0)$ & $0(0)$ & 1.000 \\
\hline Noninsulin-dependent diabetes mellitus & $13(15.5)$ & $20(23)$ & $0(0)$ & $5(38.5)$ & .072 \\
\hline Arterial hypertension & $62(73.8)$ & $65(74.7)$ & $10(100)$ & $12(92.3)$ & .153 \\
\hline Hypercholesterolemia & $53(63.1)$ & $58(66.7)$ & $8(80)$ & $9(69.2)$ & .775 \\
\hline Chronic obstructive pulmonary disease & $13(15.5)$ & $13(14.9)$ & $1(10)$ & $4(30.8)$ & .511 \\
\hline Current smoking habit & 10 (11.9) & $16(18.4)$ & $1(10)$ & $2(15.4)$ & .702 \\
\hline Preoperative serum creatinine $(\mu \mathrm{mol} / \mathrm{L})$ & $82.7(73.4-97.2)$ & $97.2(76.6-114.9)$ & $97.2(82.7-136.4)$ & $105.2(86.2-123.8)$ & .002 \\
\hline Peripheral vascular disease & $23(27.4)$ & $19(21.8)$ & $1(10)$ & $7(53.8)$ & .074 \\
\hline Left ventricular dysfunction* & $12(14.3)$ & $22(25.3)$ & $3(30)$ & $4(30.8)$ & .162 \\
\hline Left ventricular ejection fraction (\%) & $57.5(50.0-60.0)$ & $50(39.2-60)$ & $52(30-61.3)$ & $50(35-60)$ & .054 \\
\hline \multicolumn{6}{|l|}{ Preoperative medication } \\
\hline ACE inhibitor/AT-II antagonist & $48(57.1)$ & $46(52.9)$ & $7(70)$ & $11(84.6)$ & .145 \\
\hline Beta-blocker & $61(72.6)$ & $63(72.4)$ & $6(60)$ & $11(84.6)$ & .631 \\
\hline Calcium channel blocker & $22(26.2)$ & $27(31)$ & $5(50)$ & $4(30.8)$ & .444 \\
\hline HMG-CoA reductase inhibitor & $48(57.1)$ & $52(59.8)$ & $7(70)$ & $8(61.5)$ & .880 \\
\hline Diuretics & $47(56)$ & $58(66.7)$ & $8(80)$ & $11(84.6)$ & .119 \\
\hline
\end{tabular}

Values are presented as median (25th-75the percentile) or frequency (\%). CRP, C-reactive protein; RIFLE, risk injury failure end-stage renal disease classification; $A C E$, Angiotensin-converting enzyme; AT-II, angiotensin-II; HMG-CoA, 3-hydroxy-3-methyl-glutaryl-coenzyme A. *Left ventricular ejection fraction $<35 \%$. 
TABLE E7. Perioperative patient characteristics according to C-reactive protein/renal risk, injury, failure, loss of renal function, end-stage renal disease classification (CRP/RIFLE) subgroup $^{20}$

\begin{tabular}{|c|c|c|c|c|c|}
\hline Variable & $\frac{\text { CRP }(-) / \operatorname{RIFLE}(-)}{(\mathrm{n}=84 ; 43.3 \%)}$ & $\frac{\operatorname{CRP}(+) / \operatorname{RIFLE}(-)}{(\mathrm{n}=87 ; 44.8 \%)}$ & $\frac{\operatorname{CRP}(-) / \operatorname{RIFLE}(+)}{(\mathrm{n}=10 ; 5.2 \%)}$ & $\frac{\operatorname{CRP}(+) / \operatorname{RIFLE}(+)}{(\mathrm{n}=13 ; 6.7 \%)}$ & $\begin{array}{c}P \\
\text { value }\end{array}$ \\
\hline \multicolumn{6}{|l|}{ Procedures } \\
\hline CABG surgery & $15(17.9)$ & $17(19.5)$ & $1(10)$ & $3(23.1)$ & .879 \\
\hline Valvular surgery & $42(50)$ & $33(37.9)$ & $6(60)$ & $5(38.5)$ & .294 \\
\hline $\mathrm{CABG}$ and valvular surgery & $18(21.4)$ & $29(33.3)$ & $3(30)$ & $3(23.1)$ & .343 \\
\hline Thoracic aortic surgery & $9(10.7)$ & $7(8.0)$ & $0(0)$ & $1(7.7)$ & .877 \\
\hline Ventricular assist device & $0(0)$ & $1(1.1)$ & $0(0)$ & $1(7.7)$ & .224 \\
\hline Redo cardiac surgery & $20(23.8)$ & $22(25.3)$ & $4(40)$ & $5(38.5)$ & .475 \\
\hline Duration of cardiopulmonary bypass (min) & $120(93-147)$ & $119(88-148)$ & $209(150-370)$ & $147(94-207)$ & .003 \\
\hline Lowest intraoperative MAP (mm Hg) & $34(28-40)$ & $35(31-40)$ & $29.5(22.75-40.25)$ & $32(29-40)$ & .304 \\
\hline \multicolumn{6}{|l|}{$\begin{array}{l}\text { Perioperative fluid balance and } \\
\text { medication }(0-24 \mathrm{~h})\end{array}$} \\
\hline Fluid intake (mL) & $8131(6473-9694)$ & $8556(6782-9706)$ & $5556(3459-7283)$ & $6356(5587-7765)$ & .002 \\
\hline Urine output (mL) & 5135 (4271-6770) & $5490(4705-6160)$ & $3922(2806-4563)$ & $3850(2820-4278)$ & $<.001$ \\
\hline Packed red blood cells (mL) & $250(0-500)$ & $500(0-750)$ & $750(0-2312)$ & $750(375-2250)$ & .022 \\
\hline Drain output (mL) & $510(371-700)$ & $450(350-700)$ & $738(451-2038)$ & $1070(610-1750)$ & .009 \\
\hline Fluid balance (mL) & $2658(1578-3814)$ & $2832(1566-4542)$ & 1314 ( -906 to 4292$)$ & $2696(1451-4257)$ & .342 \\
\hline Furosemide (mg) & $20(0-40)$ & $30(10-50)$ & $0(0-40)$ & $60(30-175)$ & $<.001$ \\
\hline \multicolumn{6}{|l|}{ Serum CRP (mg/L) } \\
\hline $0 \mathrm{~h}$ & $0.9(0.4-1.4)$ & $5.1(3.3-12.1)$ & $1.0(0.7-1.7)$ & $8.7(2.9-11.8)$ & $<.001$ \\
\hline $6 \mathrm{~h}$ & $1.6(1.1-2.3)$ & $6.3(4.4-11.1)$ & $1.3(0.8-2.4)$ & $5.9(3.8-11.0)$ & $<.001$ \\
\hline $24 \mathrm{~h}$ & $56.1(38-82.4)$ & $77.5(60.8-106.8)$ & $43.6(28.5-70.8)$ & $81.1(67.2-124.6)$ & $<.001$ \\
\hline RIFLE stage & & & & & .275 \\
\hline Risk & & & $4(40)$ & $9(69.2)$ & \\
\hline Injury & & & $3(30)$ & $3(23.1)$ & \\
\hline Failure & & & $3(30)$ & $1(7.7)$ & \\
\hline
\end{tabular}

Values are presented as median (25th-75th percentile) or frequency (\%). CRP, C-reactive protein; RIFLE, risk injury failure end-stage renal disease classification; $C A B G$, coronary artery bypass graft; $M A P$, mean arterial pressure. 
TABLE E8. Preoperative patient characteristics according to proteinuria/renal risk, injury, failure, loss of renal function, end-stage renal disease classification (Prot/RIFLE) subgroup ${ }^{20}$

\begin{tabular}{|c|c|c|c|c|c|}
\hline Variable & $\frac{\operatorname{Prot}(-) / \text { RIFLE }(-)}{(n=80 ; 40.2 \%)}$ & $\frac{\operatorname{Prot}(+) / \text { RIFLE }(-)}{(n=95 ; 47.5 \%)}$ & $\frac{\operatorname{Prot}(-) / \text { RIFLE }(+)}{(n=10 ; 5.0 \%)}$ & $\frac{\operatorname{Prot}(+) / \operatorname{RIFLE}(+)}{(\mathrm{n}=14 ; 7.0 \%)}$ & $P$ value \\
\hline Age (y) & $69(58.3-74.5)$ & $67.0(59.5-74.8)$ & $73.0(70.5-77.3)$ & $71.5(66.5-77)$ & .097 \\
\hline Female sex & $27(33.8)$ & $23(24.2)$ & $2(20.0)$ & $6(42.9)$ & .305 \\
\hline Body mass index & $25.9(23.4-29.4)$ & $27.0(24.2-29.2)$ & $27.5(25.6-32.8)$ & $26.9(24.5-31.2)$ & .281 \\
\hline Insulin-dependent diabetes mellitus & $2(2.5)$ & $6(6.3)$ & $0(0)$ & $0(0)$ & .625 \\
\hline Noninsulin-dependent diabetes mellitus & $15(18.8)$ & $19(20.0)$ & $3(30.0)$ & $2(14.3)$ & .801 \\
\hline Arterial hypertension & $59(73.8)$ & $70(73.7)$ & $9(90.0)$ & $14(100)$ & .086 \\
\hline Hypercholesterolemia & $48(60.0)$ & $64(67.4)$ & $6(60.0)$ & $11(78.6)$ & .510 \\
\hline Chronic obstructive pulmonary disease & $15(18.8)$ & $12(12.6)$ & $2(20.0)$ & $3(21.4)$ & .556 \\
\hline Current smoking habit & $12(15.0)$ & $15(15.8)$ & $1(10)$ & $3(21.4)$ & .898 \\
\hline Preoperative serum creatinine $(\mu \mathrm{mol} / \mathrm{L})$ & $88.4(77.8-106.1)$ & $86.2(77.1-106.1)$ & $96.8(87.3-126.0)$ & $97.2(72.9-123.8)$ & .248 \\
\hline Peripheral vascular disease & $17(21.3)$ & $27(28.4)$ & $4(40.0)$ & $4(28.6)$ & .457 \\
\hline Left ventricular dysfunction* & $21(26.3)$ & $13(13.7)$ & $4(40.0)$ & $3(21.4)$ & .065 \\
\hline Left ventricular ejection fraction (\%) & $50.0(37.0-60.0)$ & $55.0(45.0-60.0)$ & $50.0(23.8-60.0)$ & $55.0(41.3-60.0)$ & .346 \\
\hline \multicolumn{6}{|l|}{ Preoperative medication } \\
\hline ACE inhibitor/AT-II antagonist & $42(52.5)$ & $54(56.8)$ & $8(80.0)$ & $10(71.4)$ & 260 \\
\hline Beta-blocker & $61(76.3)$ & $66(69.5)$ & $8(80.0)$ & $10(71.4)$ & .774 \\
\hline Calcium channel blocker & $22(27.5)$ & $28(29.5)$ & $5(50.0)$ & $5(37.7)$ & 472 \\
\hline HMG-CoA reductase inhibitor & $42(52.5)$ & $58(61.1)$ & $5(50.0)$ & $10(71.4)$ & 449 \\
\hline Diuretics & $57(71.3)$ & $51(53.7)$ & $7(70.0)$ & $13(92.9)$ & .007 \\
\hline
\end{tabular}

Values are presented as median (25th-75th percentile) or frequency (\%). Prot, Proteinuria; RIFLE, risk injury failure end-stage renal disease classification; $A C E$, angiotensin-converting enzyme; AT-II, angiotensin-II; HMG-CoA, 3-hydroxy-3-methyl-glutaryl-coenzyme A. *Left ventricular ejection fraction $<35 \%$.

TABLE E9. Perioperative patient characteristics according to proteinuria/renal risk, injury, failure, loss of renal function, end-stage renal disease classification (Prot/RIFLE) subgroup ${ }^{20}$

\begin{tabular}{|c|c|c|c|c|c|}
\hline Variable & $\frac{\operatorname{Prot}(-) / \operatorname{RIFLE}(-)}{(\mathrm{n}=80 ; 40.2 \%)}$ & $\frac{\operatorname{Prot}(+) / \operatorname{RIFLE}(-)}{(\mathrm{n}=95 ; 47.5 \%)}$ & $\frac{\operatorname{Prot}(-) / \operatorname{RIFLE}(+)}{(\mathbf{n}=10 ; 5.0 \%)}$ & $\frac{\operatorname{Prot}(+) / \operatorname{RIFLE}(+)}{(\mathbf{n}=14 ; \mathbf{7 . 0} \%)}$ & $\begin{array}{c}P \\
\text { value }\end{array}$ \\
\hline \multicolumn{6}{|l|}{ Procedures } \\
\hline CABG surgery & $16(20.0)$ & $15(15.8)$ & $3(30.0)$ & $1(7.1)$ & .443 \\
\hline Valvular surgery & $39(48.8)$ & $39(41.1)$ & $3(30.0)$ & $9(64.3)$ & .265 \\
\hline CABG and valvular surgery & $19(23.8)$ & $29(30.5)$ & $2(20.0)$ & $4(28.6)$ & .774 \\
\hline Thoracic aortic surgery & $5(6.3)$ & $12(12.6)$ & $1(10.0)$ & $0(0)$ & .324 \\
\hline Ventricular assist device & $1(1.3)$ & $0(0)$ & $1(10.0)$ & $0(0)$ & .054 \\
\hline Redo cardiac surgery & $19(23.8)$ & $23(24.2)$ & $2(20)$ & $8(57.1)$ & .077 \\
\hline Duration of cardiopulmonary bypass (min) & $116(88-137)$ & $127(94-184)$ & $144(93-256)$ & $198(149-300)$ & $<.001$ \\
\hline Lowest intraoperative MAP (mm Hg) & $37(31-41)$ & $34(28-39)$ & $31(28.25-34)$ & $29.5(23.75-41)$ & .058 \\
\hline \multicolumn{6}{|l|}{ Perioperative fluid balance and medication $(0-24 \mathrm{~h})$} \\
\hline Fluid intake $(\mathrm{mL})$ & 8709 (7343-9706) & $7540(6062-9747)$ & $5984(4289-7325)$ & $6584(5282-8782)$ & $<.001$ \\
\hline Urine output $(\mathrm{mL})$ & $5400(4639-6433)$ & $5377(4433-6488)$ & $4110(2805-4246)$ & $3836(2838-4773)$ & $<.001$ \\
\hline Packed red blood cells $(\mathrm{mL})$ & $250(0-500.0)$ & $500(0-750)$ & $1000(375-2500)$ & $525(0-2000)$ & .013 \\
\hline Drain output $(\mathrm{mL})$ & $500(300-700)$ & $485(375-700)$ & $1135(542-1625)$ & $813(509-2038)$ & .006 \\
\hline Fluid balance (mL) & $2982(2119-4476)$ & $2387(1162-3709)$ & $2124(999-3509)$ & $2711(-118$ to 4842$)$ & .091 \\
\hline Furosemide (mg) & $20.0(10.0-40.0)$ & $20.0(0-47.5)$ & $70.0(32.5-2180)$ & $55.0(20.0-150.0)$ & $<.001$ \\
\hline RIFLE stage & & & & & .461 \\
\hline Risk & & & $7(70.0)$ & $6(42.9)$ & \\
\hline Injury & & & $2(20.0)$ & $5(35.7)$ & \\
\hline Failure & & & $1(10.0)$ & $3(21.4)$ & \\
\hline
\end{tabular}

Values are presented as denote median (25th-75th percentile) or frequency (\%). Prot, Proteinuria; RIFLE, risk injury failure end-stage renal disease classification $C A B G$, coronary artery bypass graft; $M A P$, mean arterial pressure. 
TABLE E10. Area under the receiver operator characteristic (AUC-ROC) curve for acute kidney injury as end point

\begin{tabular}{lcc}
\hline Biomarker & AUC-ROC $(\mathbf{9 5} \%$ confidence interval $)$ & $\boldsymbol{P}$ value \\
\hline NGAL & $0.724(0.612-0.836)$ & $<.001$ \\
Midkine & $0.719(0.598-0.840)$ & .001 \\
Interleukin 6 & $0.732(0.625-0.839)$ & .001 \\
CRP & $0.501(0.369-0.632)$ & .992 \\
\hline
\end{tabular}

$A U C-R O C$, Area under the receiver operator characteristic curve; $N G A L$, neutrophil gelatinase-associated lipocalin; $C R P, \mathrm{C}$-reactive protein
TABLE E12. Cox regression analysis for in-hospital mortality or renal replacement therapy as dependent end point

\begin{tabular}{|c|c|c|}
\hline Variables in the equation & $\begin{array}{c}\text { Hazard ratio } \\
(95 \% \text { confidence } \\
\text { interval) }\end{array}$ & $\begin{array}{c}P \\
\text { value }\end{array}$ \\
\hline \multicolumn{3}{|l|}{ Model 1: NGAL/RIFLE } \\
\hline Age & $1.04(0.98-1.10)$ & .179 \\
\hline Previous cardiac surgery & $1.56(0.52-4.87)$ & .412 \\
\hline CABG vs concomitant & $1.00(0.36-2.82)$ & 1.000 \\
\hline Perioperative fluid balance & $1.00(0.99-1.00)$ & 1.000 \\
\hline NGAL/RIFLE group allocation & $3.54(2.36-5.31)$ & $<.001$ \\
\hline \multicolumn{3}{|l|}{ Model 2: Midkine/RIFLE } \\
\hline Age & $1.04(0.98-1.09)$ & .183 \\
\hline Previous cardiac surgery & $1.19(0.41-3.49)$ & .740 \\
\hline CABG vs concomitant & $1.13(0.41-3.14)$ & .811 \\
\hline Perioperative fluid balance & $1.00(0.99-1.00)$ & .177 \\
\hline Midkine/RIFLE group allocation & $3.43(2.31-5.09)$ & $<.001$ \\
\hline \multicolumn{3}{|l|}{ Model 3: IL-6/RIFLE } \\
\hline Age & $1.06(1.00-1.12)$ & .064 \\
\hline Previous cardiac surgery & $1.68(0.46-6.10)$ & .433 \\
\hline CABG vs concomitant & $0.58(0.19-1.78)$ & .341 \\
\hline Perioperative fluid balance & $1.00(0.99-1.00)$ & .310 \\
\hline IL-6/RIFLE group allocation & $3.72(2.34-5.92)$ & $<.001$ \\
\hline \multicolumn{3}{|l|}{ Model 4: Proteinuria/RIFLE } \\
\hline Age & $1.05(0.99-1.10)$ & .089 \\
\hline Previous cardiac surgery & $2.07(0.65-6.59)$ & .219 \\
\hline CABG vs concomitant & $0.56(0.20-1.56)$ & .269 \\
\hline Perioperative fluid balance & $0.99(0.99-1.00)$ & .014 \\
\hline Proteinuria/RIFLE group allocation & $4.38(2.66-7.22)$ & $<.001$ \\
\hline \multicolumn{3}{|l|}{ Model 5: CRP/RIFLE } \\
\hline Age & $1.05(1.00-1.11)$ & .052 \\
\hline Previous cardiac surgery & $1.53(0.49-4.81)$ & .467 \\
\hline CABG vs concomitant & $0.67(0.23-1.92)$ & .451 \\
\hline Perioperative fluid balance & $1.00(0.99-1.00)$ & .031 \\
\hline CRP/RIFLE group allocation & $2.92(1.90-4.49)$ & $<.001$ \\
\hline
\end{tabular}

TABLE E11. Comparison of the area under the receiver operating characteristic (ROC) curve for all biomarker combinations

\begin{tabular}{lccc}
\hline Paired biomarkers & $\begin{array}{c}\text { Difference } \\
\text { area A }- \text { area B }\end{array}$ & $\begin{array}{c}\text { Standard } \\
\text { error }\end{array}$ & $\begin{array}{c}\boldsymbol{P} \\
\text { value }\end{array}$ \\
\hline NGAL - midkine & 0 & 0.087 & 1.0 \\
NGAL - IL-6 & 0.01 & 0.0906 & .912 \\
IL-6 - midkine & 0.01 & 0.0907 & .912 \\
NGAL - CRP & 0.22 & 0.089 & .013 \\
IL-6 - CRP & 0.23 & 0.0925 & .013 \\
Midkine - CRP & 0.22 & 0.089 & .013 \\
\hline
\end{tabular}

$N G A L$, Neutrophil gelatinase-associated lipocalin; $I L-6$, interleukin 6; $C R P$, C-reactive protein. *Comparison of the area under the ROC curves for acute kidney injury as dependent end point did not show differences comparing NGAL, midkine, and IL-6 with each other. However, the area under the curve for CRP differed significantly compared with all urinary biomarkers. 
TABLE E13. Cox regression analysis for in-hospital mortality as dependent end point

\begin{tabular}{|c|c|c|}
\hline Variables in the equation & $\begin{array}{c}\text { Hazard ratio } \\
\text { (95\% confidence } \\
\text { interval) }\end{array}$ & $\begin{array}{c}P \\
\text { value }\end{array}$ \\
\hline \multicolumn{3}{|l|}{ Model 1: NGAL/RIFLE } \\
\hline Age & $1.04(0.97-1.12)$ & .271 \\
\hline Previous cardiac surgery & $1.27(0.64-2.53)$ & .501 \\
\hline CABG vs concomitant & $0.79(0.23-2.73)$ & .708 \\
\hline Perioperative fluid balance $(0-6 \mathrm{~h})$ & $0.99(0.99-1.00)$ & .035 \\
\hline NGAL/RIFLE group allocation & $3.07(1.91-4.93)$ & $<.001$ \\
\hline \multicolumn{3}{|l|}{ Model 2: Midkine/RIFLE } \\
\hline Age & $1.04(0.97-1.11)$ & .324 \\
\hline Previous cardiac surgery & $1.19(0.61-2.34)$ & .615 \\
\hline CABG vs concomitant & $0.95(0.28-3.24)$ & .931 \\
\hline Perioperative fluid balance $(0-6 \mathrm{~h})$ & $0.99(0.99-1.00)$ & .044 \\
\hline Midkine/RIFLE group allocation & $3.10(1.94-4.96)$ & $<.001$ \\
\hline \multicolumn{3}{|l|}{ Model 3: IL-6/RIFLE } \\
\hline Age & $1.06(0.98-1.15)$ & .138 \\
\hline Previous cardiac surgery & $1.83(0.64-5.22)$ & .261 \\
\hline CABG vs concomitant & $0.50(0.12-2.12)$ & .347 \\
\hline Perioperative fluid balance $(0-6 \mathrm{~h})$ & $1.00(0.99-1.00)$ & .154 \\
\hline IL-6/RIFLE group allocation & $2.81(1.63-4.84)$ & $<.001$ \\
\hline \multicolumn{3}{|l|}{ Model 4: Proteinuria/RIFLE } \\
\hline Age & $1.05(0.98-1.13)$ & .190 \\
\hline Previous cardiac surgery & $1.43(0.70-2.94)$ & .329 \\
\hline CABG vs concomitant & $0.46(0.13-1.63)$ & .231 \\
\hline Perioperative fluid balance $(0-6 \mathrm{~h})$ & $0.99(0.99-1.00)$ & .008 \\
\hline Proteinuria/RIFLE group allocation & $3.93(2.15-7.20)$ & $<.001$ \\
\hline \multicolumn{3}{|l|}{ Model 5: CRP/RIFLE } \\
\hline Age & $1.07(0.99-1.14)$ & .083 \\
\hline Previous cardiac surgery & $1.39(0.64-3.06)$ & .408 \\
\hline CABG vs concomitant & $0.58(0.16-2.11)$ & .408 \\
\hline Perioperative fluid balance $(0-6 \mathrm{~h})$ & $0.99(0.99-1.00)$ & .012 \\
\hline CRP/RIFLE group allocation & $2.44(1.45-4.10)$ & .001 \\
\hline
\end{tabular}

$N G A L$, Neutrophil gelatinase-associated lipocalin; RIFLE, renal risk, injury, failure, loss of renal function, end-stage renal disease classification ${ }^{20} ; C A B G$, coronary artery bypass graft; $I L-6$, interleukin-6; $C R P$, C-reactive protein.
TABLE E14. Cox regression analysis for major adverse kidney events (MAKE) as dependent end point

\begin{tabular}{|c|c|c|}
\hline Variables in the equation & $\begin{array}{c}\text { Hazard ratio } \\
\text { (95\% confidence } \\
\text { interval) }\end{array}$ & $\begin{array}{c}P \\
\text { value }\end{array}$ \\
\hline \multicolumn{3}{|l|}{ Model 1: NGAL/RIFLE } \\
\hline Age & $1.04(0.99-1.10)$ & .102 \\
\hline Previous cardiac surgery & $1.39(0.49-3.96)$ & .541 \\
\hline CABG vs concomitant & $1.16(0.43-3.10)$ & .768 \\
\hline Perioperative fluid balance & $1.00(0.99-1.00)$ & .150 \\
\hline NGAL/RIFLE group allocation & $3.71(2.52-5.46)$ & $<.001$ \\
\hline \multicolumn{3}{|l|}{ Model 2: Midkine/RIFLE } \\
\hline Age & $1.05(0.99-1.01)$ & .083 \\
\hline Previous cardiac surgery & $0.96(0.36-2.55)$ & .936 \\
\hline CABG vs concomitant & $1.31(0.49-3.44)$ & .584 \\
\hline Perioperative fluid balance & $1.00(0.99-1.00)$ & .202 \\
\hline Midkine/RIFLE group allocation & $3.75(2.54-5.54)$ & $<.001$ \\
\hline \multicolumn{3}{|l|}{ Model 3: IL-6/RIFLE } \\
\hline Age & $1.06(1.00-1.12)$ & .035 \\
\hline Previous cardiac surgery & $1.31(0.41-4.19)$ & .650 \\
\hline CABG vs concomitant & $0.63(0.22-1.84)$ & .398 \\
\hline Perioperative fluid balance & $1.00(0.99-1.00)$ & .284 \\
\hline IL-6/RIFLE group allocation & $4.16(2.61-6.64)$ & $<.001$ \\
\hline \multicolumn{3}{|l|}{ Model 4: Proteinuria/RIFLE } \\
\hline Age & $1.05(1.00-1.11)$ & .047 \\
\hline Previous cardiac surgery & $1.81(0.61-5.38)$ & .286 \\
\hline CABG vs concomitant & $0.64(0.24-1.69)$ & .369 \\
\hline Perioperative fluid balance & $0.99(0.99-1.00)$ & .014 \\
\hline Proteinuria/RIFLE group allocation & $4.79(2.94-7.80)$ & $<.001$ \\
\hline \multicolumn{3}{|l|}{ Model 5: CRP/RIFLE } \\
\hline Age & $1.06(1.00-1.11)$ & .036 \\
\hline Previous cardiac surgery & $1.09(0.40-2.97)$ & .861 \\
\hline CABG vs concomitant & $0.78(0.28-2.16)$ & .633 \\
\hline Perioperative fluid balance & $1.00(0.99-1.00)$ & .049 \\
\hline CRP/RIFLE group allocation & $3.33(2.17-5.11)$ & $<.001$ \\
\hline
\end{tabular}

$N G A L$, Neutrophil gelatinase-associated lipocalin; RIFLE, renal risk, injury, failure, loss of renal function, end-stage renal disease classification ${ }^{20} ; C A B G$, coronary artery bypass graft; $I L-6$, interleukin 6 ; $C R P$, C-reactive protein. 
TABLE E15. Cox regression analysis for in-hospital adverse events (primary and secondary end points) according to biomarker/renal risk, injury, failure, loss of renal function, end stage renal disease classification (RIFLE) groups compared with reference (biomarker[-]/RIFLE[-] $)^{20}$

\begin{tabular}{|c|c|c|}
\hline Groups compared & Hazard ratio ( $95 \%$ confidence interval) & $P$ value \\
\hline \multicolumn{3}{|l|}{ In-hospital mortality or RRT as dependent end point } \\
\hline Midkine(+)/RIFLE(-) vs midkine(-)/RIFLE(-) & $2.33(0.53-10.35)$ & .265 \\
\hline Midkine(-)/RIFLE(+) vs midkine(-)/RIFLE(-) & $6.27(0.91-43.03)$ & .062 \\
\hline Midkine $(+) / \operatorname{RIFLE}(+)$ vs midkine $(-) / \operatorname{RIFLE}(-)$ & $67.59(17.32-263.78)$ & $<.001$ \\
\hline IL-6(+)/RIFLE(-) vs IL-6(-)/RIFLE(-) & $6.27(1.12-35.21)$ & .037 \\
\hline IL-6(-)/RIFLE(+) vs IL-6(-)/RIFLE(-) & $22.19(1.2-417.38)$ & .038 \\
\hline IL-6(+)/RIFLE(+) vs IL-6(-)/RIFLE(-) & $91.08(10.71-774.30)$ & $<.001$ \\
\hline $\operatorname{Prot}(+) / \operatorname{RIFLE}(-)$ vs Prot(-)/RIFLE(-) & $2.15(0.41-11.27)$ & .366 \\
\hline $\operatorname{Prot}(-) / \operatorname{RIFLE}(+)$ vs Prot(-)/RIFLE(-) & $29.50(3.11-280.01)$ & .003 \\
\hline $\operatorname{Prot}(+) / \operatorname{RIFLE}(+)$ vs $\operatorname{Prot}(-) / \operatorname{RIFLE}(-)$ & $66.63(11.82-375.48)$ & $<.001$ \\
\hline $\mathrm{CRP}(+) / \operatorname{RIFLE}(-)$ vs $\operatorname{CRP}(-) / \operatorname{RIFLE}(-)$ & $0.92(0.21-4.01)$ & .913 \\
\hline CRP(-)/RIFLE(+) vs CRP(-)/RIFLE(-) & $13.39(3.16-56.78)$ & $<.001$ \\
\hline $\mathrm{CRP}(+) / \operatorname{RIFLE}(+)$ vs CRP(-)/RIFLE(-) & $11.41(2.86-45.42)$ & .001 \\
\hline \multicolumn{3}{|l|}{ In-hospital mortality as dependent end point } \\
\hline Midkine(+)/RIFLE(-) vs midkine(-)/RIFLE(-) & $4.27(0.66-27.56)$ & .128 \\
\hline Midkine $(-) / \operatorname{RIFLE}(+)$ vs midkine $(-) / \operatorname{RIFLE}(-)$ & $6.65(0.46-95.73)$ & .164 \\
\hline Midkine $(+) / \operatorname{RIFLE}(+)$ vs midkine $(-) / \operatorname{RIFLE}(-)$ & $42.48(7.74-233.06)$ & $<.001$ \\
\hline IL-6(+)/RIFLE(-) vs IL-6(-)/RIFLE(-) & $5.41(0.58-50.26)$ & .137 \\
\hline IL-6(-)/RIFLE(+) vs IL-6(-)/RIFLE(-) & $0.99(0.99-1.00)$ & .994 \\
\hline IL-6(+)/RIFLE(+) vs IL-6(-)/RIFLE(-) & $159.87(3.39-7532.42)$ & .01 \\
\hline $\operatorname{Prot}(+) / \operatorname{RIFLE}(-)$ vs Prot(-)/RIFLE(-) & $4.03(0.43-37.79)$ & .222 \\
\hline $\operatorname{Prot}(-) / \operatorname{RIFLE}(+)$ vs Prot(-)/RIFLE(-) & $67.57(2.47-1850.67)$ & .013 \\
\hline $\operatorname{Prot}(+) / \operatorname{RIFLE}(+)$ vs Prot(-)/RIFLE(-) & $62.92(4.95-800.1)$ & .001 \\
\hline $\mathrm{CRP}(+) / \operatorname{RIFLE}(-)$ vs CRP(-)/RIFLE(-) & $0.51(0.08-3.21)$ & .476 \\
\hline $\mathrm{CRP}(-) / \operatorname{RIFLE}(+)$ vs CRP(-)/RIFLE(-) & $6.49(1.22-34.49)$ & .028 \\
\hline $\mathrm{CRP}(+) / \operatorname{RIFLE}(+)$ vs CRP(-)/RIFLE(-) & $9.69(1.88-49.90)$ & .007 \\
\hline \multicolumn{3}{|l|}{ Major adverse kidney events as dependent end point } \\
\hline Midkine(+)/RIFLE(-) vs midkine(-)/RIFLE(-) & $2.33(0.53-10.35)$ & .265 \\
\hline Midkine(-)/RIFLE(+) vs midkine(-)/RIFLE(-) & $16.03(3.07-83.82)$ & .001 \\
\hline Midkine(+)/RIFLE(+) vs midkine(-)/RIFLE(-) & $67.59(17.32-263.78)$ & $<.001$ \\
\hline IL-6(+)/RIFLE(-) vs IL-6(-)/RIFLE(-) & $6.27(1.12-35.21)$ & .037 \\
\hline IL-6(-)/RIFLE(+) vs IL-6(-)/RIFLE(-) & $45.33(2.98-689.49)$ & .006 \\
\hline $\operatorname{IL}-6(+) / \operatorname{RIFLE}(+)$ vs IL-6(-)/RIFLE(-) & $104.85(12.32-892.54)$ & $<.001$ \\
\hline $\operatorname{Prot}(+) / \operatorname{RIFLE}(-)$ vs $\operatorname{Prot}(-) / \operatorname{RIFLE}(-)$ & $2.15(0.41-11.27)$ & .366 \\
\hline $\operatorname{Prot}(-) / \operatorname{RIFLE}(+)$ vs Prot(-)/RIFLE(-) & $34.98(3.77-324.49)$ & .002 \\
\hline $\operatorname{Prot}(+) / \operatorname{RIFLE}(+)$ vs Prot(-)/RIFLE(-) & $78.41(13.85-444.0)$ & $<.001$ \\
\hline $\mathrm{CRP}(+) / \operatorname{RIFLE}(-)$ vs CRP(-)/RIFLE(-) & $0.92(0.21-4.01)$ & .913 \\
\hline CRP(-)/RIFLE(+) vs CRP(-)/RIFLE(-) & $18.48(4.40-77.64)$ & $<.001$ \\
\hline $\mathrm{CRP}(+) / \operatorname{RIFLE}(+)$ vs $\operatorname{CRP}(-) / \operatorname{RIFLE}(-)$ & $13.64(3.57-52.01)$ & $<.001$ \\
\hline
\end{tabular}

Model adjusted for age, type of surgery (coronary artery bypass graft vs concomittant surgery), previous cardiac surgery, and perioperative fluid balance 0-6. RRT, Renal replacement therapy; RIFLE, renal risk, injury, failure, loss of renal function, end-stage renal disease classification ${ }^{20} ; I L-6$, interleukin-6; Prot, proteinuria; $C R P$, C-reactive protein. 
TABLE E16. Cox regression analysis for long-term overall survival (long-term outcome)

\begin{tabular}{|c|c|c|}
\hline Variables in the equation & $\begin{array}{c}\text { Hazard ratio } \\
\text { (95\% confidence } \\
\text { interval }) \\
\end{array}$ & $\begin{array}{c}P \\
\text { value } \\
\end{array}$ \\
\hline \multicolumn{3}{|l|}{ Model 1: NGAL/RIFLE ${ }^{20}$} \\
\hline NGAL/RIFLE group allocation & $2.251(1.676-3.022)$ & $<.001$ \\
\hline Preoperative serum creatinine & $1.002(0.991-1.014)$ & .690 \\
\hline Peripheral vascular disease & $0.770(0.407-1.457)$ & .421 \\
\hline Arterial hypertension & $1.544(0.670-3.557)$ & .308 \\
\hline $\begin{array}{l}\text { Myocardial infarction } \\
6 \text { mo before surgery }\end{array}$ & $1.476(0.637-3.421)$ & .363 \\
\hline \multicolumn{3}{|l|}{ Model 2: Midkine/RIFLE } \\
\hline Midkine/RIFLE group allocation & $2.154(1.655-2.804)$ & $<.001$ \\
\hline Preoperative serum creatinine & $1.002(0.990-1.013)$ & .790 \\
\hline Peripheral vascular disease & $0.799(0.397-1.479)$ & .428 \\
\hline Arterial hypertension & $1.558(0.677-3.585)$ & .297 \\
\hline $\begin{array}{l}\text { Myocardial infarction } \\
6 \text { mo before surgery }\end{array}$ & $1.388(0.534-3.610)$ & .501 \\
\hline \multicolumn{3}{|l|}{ Model 3: IL-6/RIFLE } \\
\hline IL-6/RIFLE group allocation & $2.027(1.527-2.700)$ & $<.001$ \\
\hline Preoperative serum creatinine & $1.011(1.000-1.022)$ & .058 \\
\hline Peripheral vascular disease & $0.873(0.455-1.675)$ & .682 \\
\hline Arterial hypertension & $1.509(0.653-3.486)$ & .335 \\
\hline $\begin{array}{l}\text { Myocardial infarction } \\
6 \text { mo before surgery }\end{array}$ & $1.260(0.544-2.918)$ & .589 \\
\hline \multicolumn{3}{|l|}{ Model 4: Proteinuria/RIFLE } \\
\hline Proteinuria/RIFLE group allocation & $1.721(1.266-2339)$ & .001 \\
\hline Preoperative serum creatinine & $1.005(0.994-1.016)$ & .377 \\
\hline Peripheral vascular disease & $0.789(0.415-1.497)$ & .468 \\
\hline Arterial hypertension & $1.874(0.826-4.253)$ & .133 \\
\hline $\begin{array}{l}\text { Myocardial infarction } \\
6 \text { mo before surgery }\end{array}$ & $1.470(0.639-3.384)$ & .365 \\
\hline
\end{tabular}

NGAL, Neutrophil gelatinase-associated lipocalin; RIFLE, renal risk, injury, failure, loss of renal function, end-stage renal disease classification; $I L-6$, interleukin- 6 .

TABLE E17. Measurement of agreement among biomarkers. The degree of agreement among all biomarkers allocating patients to acute kidney injury subtype groups is presented. We found moderate to substantial agreement between neutrophil gelatinase-associated lipocalin (NGAL) and midkine and fair agreement with proteinuria. However, there was only poor to slight agreement between C-reactive protein (CRP) with kidney biomarkers and proteinuria

\begin{tabular}{|c|c|c|c|c|}
\hline Paired biomarker & $\kappa$ & Standard error & Significance level & Strength of agreement \\
\hline NGAL - midkine & 0.579 & 0.060 & $<.001$ & Moderate-substantial \\
\hline NGAL - IL-6 & 0.452 & 0.060 & $<.001$ & Moderate \\
\hline IL-6 - midkine & 0.428 & 0.062 & $<.001$ & Moderate \\
\hline NGAL - proteinuria & 0.322 & 0.052 & $<.001$ & Fair \\
\hline IL- 6 - proteinuria & 0.250 & 0.061 & $<.001$ & Fair \\
\hline Midkine - proteinuria & 0.238 & 0.053 & $<.001$ & Fair \\
\hline IL-6 - CRP & 0.180 & 0.065 & .001 & Slight \\
\hline NGAL - CRP & 0.144 & 0.058 & .003 & Slight \\
\hline Proteinuria - CRP & 0.018 & 0.061 & .738 & Poor-slight \\
\hline Midkine - CRP & 0.097 & 0.056 & .032 & Poor-slight \\
\hline
\end{tabular}

Graduated on recommended basis of poor, slight, fair, moderate, substantial, and almost perfect. NGAL, Neutrophil gelatinase-associated lipocalin; $I L-6$, interleukin-6; $C R P$, C-reactive protein. *Measurement of agreement and interpretation according to recommendation. ${ }^{25}$ 